\title{
Minimal energy solutions for cooperative nonlinear Schrödinger systems
}

\author{
Rainer Mandel
}

\begin{abstract}
We prove sharp existence and nonexistence results for minimal energy solutions of the nonlinear Schrödinger system

$$
\begin{aligned}
-\Delta u+u & =|u|^{2 q-2} u+b|u|^{q-2} u|v|^{q} & & \text { in } \mathbb{R}^{n}, \\
-\Delta v+\omega^{2} v & =|v|^{2 q-2} v+b|u|^{q}|v|^{q-2} v & & \text { in } \mathbb{R}^{n}
\end{aligned}
$$

in the cooperative and subcritical case $b>0,1<q<\frac{n}{(n-2)_{+}}$. The proofs are accomplished by minimizing the Euler functional of $(1)$ over the two associated Nehari manifolds. In the special case $1<q<2$ we find that a positive solution of (1) with minimal energy among all nontrivial solutions exists if and only if $b>0$.
\end{abstract}

Mathematics Subject Classification (2010). Primary: 35J50, 35J57.

Keywords. Variational methods for elliptic systems, Nonlinear Schrödinger systems.

\section{Introduction}

During the past ten years many authors have contributed to a better understanding of nonlinear Schrödinger systems of the type

$$
\begin{aligned}
& -\Delta u+u=|u|^{2 q-2} u+b|u|^{q-2} u|v|^{q}, \\
& -\Delta v+\omega^{2} v=|v|^{2 q-2} v+b|u|^{q}|v|^{q-2} v, \\
& \omega>0, b \in \mathbb{R}, \quad 1<q<\frac{n}{(n-2)_{+}}, \quad u, v \in H^{1}\left(\mathbb{R}^{n}\right)
\end{aligned}
$$

where the case of a cubic nonlinearity $q=2$ is of particular mathematical and physical interest. One of the most powerful methods to prove existence results for nontrivial low energy solutions of (2) is the constrained minimization method. Exploiting the variational structure of the nonlinear Schrödinger system (2) the existence of a nontrivial solution is proved by minimizing the associated Euler functional

$$
I(u, v)=\frac{1}{2}\left(\|u\|^{2}+\|v\|_{\omega}^{2}\right)-\frac{1}{2 q}\left(\|u\|_{2 q}^{2 q}+\|v\|_{2 q}^{2 q}+2 b\|u v\|_{q}^{q}\right)
$$


over the so-called Nehari set or Nehari manifold. For a definition of the norms appearing in (3) we refer to Sect. 2. In the context of the nonlinear Schrödinger system (2) there are two natural choices for the Nehari set which we will denote by $\mathcal{N}_{b}, \mathcal{M}_{b}$. In this paper we investigate the minimization problems

$$
c_{b}:=\inf _{\mathcal{N}_{b}} I, \quad \kappa_{b}:=\inf _{\mathcal{M}_{b}} I
$$

in order to improve known existence and nonexistence results for nontrivial minimal energy solutions of (2). Here, according to the definition of $\mathcal{N}_{b}, \mathcal{M}_{b}$, minimality will either refer to the class of nontrivial finite energy solutions $(u, v) \neq(0,0)$ or to the class of fully nontrivial finite energy solutions $(u, v)$ satisfying both $u \neq 0$ and $v \neq 0$.

First we consider the minimization problem over the Nehari set $\mathcal{N}_{b}$ given by

$$
\begin{array}{r}
\mathcal{N}_{b}=\left\{(u, v) \in H^{1}\left(\mathbb{R}^{n}\right) \times H^{1}\left(\mathbb{R}^{n}\right):(u, v) \neq(0,0),\right. \\
\left.\|u\|^{2}+\|v\|_{\omega}^{2}=\|u\|_{2 q}^{2 q}+\|v\|_{2 q}^{2 q}+2 b\|u v\|_{q}^{q}\right\} .
\end{array}
$$

The Lagrange multiplier rule implies that every minimizer of $\left.I\right|_{\mathcal{N}_{b}}$ is a nontrivial solution of (2) having minimal energy among all nontrivial finite energy solutions. Following [11] we call such a solution a ground state and it is called a vector ground state in case both components are nontrivial. The existence of ground states for all $b \geq 0, \omega>0$ and all subcritical exponents $1<q<\frac{n}{(n-2)_{+}}$ has been proved by Maia, Montefusco, Pellacci (cf. Theorem 2.1 in [11]) so that the main problem is to find sufficient or necessary conditions for these ground states to be vector ground states. In the case of a cubic nonlinearity $q=2$ sufficient conditions have been found by Ambrosetti, Colorado [1] and de Figueiredo, Lopes [5] whereas the general case $1<q<\frac{n}{(n-2)_{+}}$was investigated in [11]. More details concerning these results will be provided later, see Remark 1. Our contribution to this problem is the proof of a sharp condition for existence and nonexistence of vector ground states. Denoting the uniquely determined positive finite energy solution of $-\Delta u+u=u^{2 q-1}$ in $\mathbb{R}^{n}$ by $u_{0}$ and setting $\hat{c}_{0}:=\left\|u_{0}\right\|\left\|u_{0}\right\|_{2 q}^{-1}$ we will show that for $\omega \geq 1$ the value

$$
b(\omega, q, n)=\inf \left\{\frac{\hat{c}_{0}^{-2 q}\left(\|u\|^{2}+\|v\|_{\omega}^{2}\right)^{q}-\|u\|_{2 q}^{2 q}-\|v\|_{2 q}^{2 q}}{2\|u v\|_{q}^{q}}: u, v \in H^{1}\left(\mathbb{R}^{n}\right)\right\}
$$

defines a threshold for existence or nonexistence of vector ground states.

Theorem 1. Let $n \in \mathbb{N}, 1<q<\frac{n}{(n-2)_{+}}$and $\omega \geq 1$.

(i) If $b<b(\omega, q, n)$ then $c_{b}=I\left(u_{0}, 0\right)$ and (2) has no vector ground states.

(ii) If $b>b(\omega, q, n)$ then $c_{b}<I\left(u_{0}, 0\right)$ and (2) has a vector ground state.

Notice that the assumption $\omega \geq 1$ is not restrictive given the fact that $(u, v)$ solves $(2)$ with parameter $\omega^{-1}$ if and only if $\left(\omega^{1 /(q-1)} v(\omega \cdot), \omega^{1 /(q-1)} u(\omega \cdot)\right)$ solves (2) with parameter $\omega$. Accordingly the threshold value for $\omega \in(0,1)$ is given by $b\left(\omega^{-1}, q, n\right)$. The borderline case $b=b(\omega, q, n)$ appears to be more complicated and our results and open questions related to this issue will be 
addressed in Appendix B. In the following two Lemmas we estimate the value $b(\omega, q, n)$ from above and below in order to get explicit sufficient respectively necessary conditions for the existence of a vector ground state.

Lemma 1. Let $n \in \mathbb{N}, 1<q<\frac{n}{(n-2)_{+}}$and $\omega \geq 1$. Then the following holds:

(i) In case $1<q<2$ we have $b(\omega, q, n) \geq 0$.

(ii) In case $q \geq 2$ we have $b(\omega, q, n) \geq \omega^{\frac{2 q-n(q-1)}{2}}\left(2^{q-1}-1\right)$.

In the next Lemma we will use the symbol $\bar{\mu}(\omega, n)$ to denote the reciprocal of the largest eigenvalue of the compact operator $\left(-\Delta+\omega^{2}\right)^{-1}\left(u_{0}^{2} \cdot\right)$ which maps $H^{1}\left(\mathbb{R}^{n}\right)$ to itself. Let us mention that this value equals $\Lambda^{\prime}$ from [1] and $\lambda_{1}^{-1}\left(\omega^{2}\right)$ from $[5]$.

Lemma 2. Let $n \in \mathbb{N}, 1<q<\frac{n}{(n-2)_{+}}$and $\omega \geq 1$. Then the following estimates hold:

(i) In case $1<q<2$ we have $b(\omega, q, n) \leq 0$.

(ii) In case $q=2$ we have $b(\omega, 2, n) \leq \bar{\mu}(\omega, n)$, in particular $b(\omega, 2,1) \leq$ $\frac{\omega(\omega+1)}{2}$ and $b(\omega, 2, n) \leq \frac{4-n}{4} \omega^{2}+\frac{n}{4}$ for $n \in\{2,3\}$. In case $n=3, \omega^{2}>5$ we moreover have

$$
\begin{aligned}
b(\omega, 2, n) \leq & \frac{\omega\left(1+\omega^{2}-\sqrt{1+3 \omega^{2}}\right)^{\frac{1}{2}}\left(-1+3 \omega^{2}-\sqrt{1+3 \omega^{2}}\right)^{\frac{3}{2}}}{4 \omega^{2}\left(-1+\omega^{2}-\sqrt{1+3 \omega^{2}}\right)} \\
& +\frac{-5 \omega^{4}-\omega^{2}-2+2\left(\omega^{2}-1\right) \sqrt{1+3 \omega^{2}}}{4 \omega^{2}\left(-1+\omega^{2}-\sqrt{1+3 \omega^{2}}\right)} .
\end{aligned}
$$

(iii) In case $q>2$ we have

$$
\begin{array}{ll}
b(\omega, q, n) \leq\left(2^{q-1}-\frac{1}{2}\right) \omega^{\frac{q+2}{2}}-\frac{1}{2} \omega^{-\frac{q}{2}} & (n=1), \\
b(\omega, q, n) \leq\left(1+\omega^{2}\right)^{\frac{2 q-n(q-1)}{2}} 2^{\frac{n(q-1)-2}{2}}-1 & (n=2,3) .
\end{array}
$$

In particular Lemma 1 (i) and Lemma 2 (i) imply $b(\omega, q, n)=0$ for all $q \in(1,2)$. Moreover we will show in Appendix B that for $b=0$ vector ground states do not exist so that Theorem 1 yields the following result.

Corollary 1. Let $n \in \mathbb{N}, 1<q<\frac{n}{(n-2)_{+}}, \omega \geq 1$ and $q \in(1,2)$. Then a vector ground state of (2) exists if and only if $b>0$.

\section{Remark 1.}

(a) Our estimates for $\omega=1$ are sharp and yield $b(1, q, n)=0$ for $q \in(1,2)$ and $b(1, q, n)=2^{q-1}-1$ for $q \geq 2$ in concordance with [11]. The corresponding vector ground states are given in Appendix A.

(b) Lemma 1 implies that $b(\cdot, q, n)$ is bounded from below by a coercive function whenever $q \geq 2$. This property of the function $b(\cdot, q, n)$ has only been known for $q=2$, see Theorem 2.9 in [11]. Moreover, in the special case $n=1$ our upper estimate $b(\omega, q, n)=O\left(\omega^{(q+2) / 2}\right)$ is asymptotically better than the estimate $b(\omega, q, n)=O\left(\omega^{q+1}\right)$ from Theorem 2.3 in [11].

(c) In the case of a cubic nonlinearity $q=2$ the existence of a vector ground state for $b>\bar{\mu}(\omega, n)$ was proved by Ambrosetti, Colorado when $n \in\{2,3\}$ and by de Figueiredo, Lopes in the one-dimensional case, cf. Theorem 2 
in [1] and Theorem 1.4 in [5]. Therefore the estimate (6) represents the only new upper bound in Lemma 2 (ii) which, however, raises an interesting question which we address in part (d). The necessary condition $b \geq \omega^{(4-n) / 2}$ from Lemma 1 (ii) goes back to [11], Theorem 2.9.

(d) At least for $n \in\{1,2\}$ we can not exclude $b(\omega, 2, n)=\bar{\mu}(\omega, n)$. In view of the equality $b(1,2, n)=\bar{\mu}(1, n)=1$ for all $n \in\{1,2,3\}$ it would be interesting to know for which parameters $\omega>1, n \in\{1,2,3\}$ this equation holds. We want to stress that the upper bound (6) implies $b(\omega, 2,3)=O(\omega)$ as $\omega \rightarrow \infty$ so that this equation can not hold when $n=3$ and $\omega$ is sufficiently large because of the inequality $\bar{\mu}(\omega, 3) \geq\left\|u_{0}\right\|_{\infty}^{-2} \omega^{2}$ which we will prove in (18).

Theorem 1 raises the question whether positive solutions of (2) with low energy also exist for coupling parameters $b<b(\omega, q, n)$. Lin and Wei $[9,10]$ and Sirakov [14] proved the existence of such solutions when $q=2$ and $b \in[0,1)$ is small. They considered the minimization problem

$$
\inf _{\mathcal{M}_{b}} I
$$

where the Nehari set $\mathcal{M}_{b}$ is given by

$$
\begin{aligned}
\mathcal{M}_{b}=\{(u, v) & \in H^{1}\left(\mathbb{R}^{n}\right) \times H^{1}\left(\mathbb{R}^{n}\right): u, v \neq 0, \\
\|u\|^{2} & \left.=\|u\|_{2 q}^{2 q}+b\|u v\|_{q}^{q}, \quad\|v\|_{\omega}^{2}=\|v\|_{2 q}^{2 q}+b\|u v\|_{q}^{q}\right\} .
\end{aligned}
$$

This set contains all fully nontrivial solutions of (2) and $\mathcal{M}_{b} \subset \mathcal{N}_{b}$ implies

$$
\kappa_{b} \geq c_{b} \quad(b \in \mathbb{R}) \quad \text { for } \quad \kappa_{b}=\inf _{\mathcal{M}_{b}} I, \quad c_{b}=\inf _{\mathcal{N}_{b}} I .
$$

We will investigate the problem (7) for all exponents $q \in\left(1, \frac{n}{(n-2)_{+}}\right)$. As in the case of the minimization problem for $\left.I\right|_{\mathcal{N}_{b}}$ we will obtain that minimizers of $\left.I\right|_{\mathcal{M}_{b}}$ are solutions of (2). By definition of $\mathcal{M}_{b}$ such a solution is fully nontrivial and has minimal energy among all fully nontrivial solutions. First we show that me may restrict ourselves to the case $0 \leq b \leq b(\omega, q, n)$ by proving that in any other case the problem (7) is either trivially solvable or unsolvable. Indeed, for $b>b(\omega, q, n)$ the solutions of the minimization problem will turn out to be the vector ground states from Theorem 1 . For $b<0$ we prove the nonexistence of minimizers of $\left.I\right|_{\mathcal{M}_{b}}$ for all $1<q<\frac{n}{(n-2)_{+}}$which generalizes a result of Lin and Wei (cf. [9], Theorem 1).

Lemma 3. Let $n \in \mathbb{N}, 1<q<\frac{n}{(n-2)_{+}}$and $\omega \geq 1$.

(i) If $b<0$ then $\kappa_{b}=I\left(u_{0}, 0\right)+I\left(0, v_{0}\right)$ and $\kappa_{b}$ is not attained.

(ii) If $b>b(\omega, q, n)$ then $\kappa_{b}=c_{b}$ and $\kappa_{b}$ is attained precisely in the vector ground states of (2).

Remark 2. For negative $b$ the existence of radially symmetric low energy solutions was shown in [13] by proving the existence of a minimizer of the functional $\left.I\right|_{\mathcal{M}_{b}^{*}}$ where $\mathcal{M}_{b}^{*}=\left\{(u, v) \in \mathcal{M}_{b}: u, v\right.$ radially symmetric $\}$. In the special case $\omega=1$ further existence results for positive solutions were proved by Bartsch, Dancer, Wang [2] using global bifurcation theory and by Wei and Weth [15] 
exploiting compactness properties of $G$-symmetric functions where $G$ is a nontrivial subgroup of the orthogonal group $O(n)$.

In view of Lemma 3 and $b(\omega, q, n)=0$ for $q \in(1,2)$ we may assume $n \in\{1,2,3\}, 2 \leq q<\frac{n}{(n-2)_{+}}$. Our result for exponents $q>2$ is the following.

Theorem 2. Let $n \in\{1,2,3\}, 2<q<\frac{n}{(n-2)_{+}}, \omega \geq 1$ and $b \in[0, q-1)$. Then $\kappa_{b}>I\left(0, v_{0}\right)$ and $\kappa_{b}$ is attained at a positive solution of $(2)$.

Finally let us turn our attention to the minimization problem (7) for $q=2$. It was shown by Sirakov (cf. [14], Theorem 2 (i)) that $0 \leq b<\tilde{\mu}(\omega, n)$ is sufficient for the existence of a minimizer of $\left.I\right|_{\mathcal{M}_{b}}$ where $\left.\underline{\tilde{\mu}}(\omega, n) \in \overline{(0}, 1\right]$ is given by

$$
\underline{\tilde{\mu}}(\omega, n):=\frac{2}{\omega^{(4-n) / 2}+\omega^{(n-4) / 2}+\sqrt{\omega^{4-n}+\omega^{n-4}+2-4 \omega^{-n / 2}}} .
$$

In Theorem 3 we improve this result by showing that for $\omega>1, b \in[0,1)$ the condition $b<\mu(\omega, n)$ is necessary and sufficient for the existence of a minimizer of $\left.I\right|_{\mathcal{M}_{b}}$ where $\mu(\omega, n)$ is the reciprocal of the largest eigenvalue of the compact operator $(-\Delta+1)^{-1}\left(v_{0}^{2} \cdot\right)$ which maps $H^{1}\left(\mathbb{R}^{n}\right)$ to itself. Here, the positive function $v_{0}:=\omega u_{0}(\omega \cdot) \in H^{1}\left(\mathbb{R}^{n}\right)$ is the uniquely determined positive finite energy solution of $-\Delta v+\omega^{2} v=v^{3}$ in $\mathbb{R}^{n}$. The unique positive normalized first eigenfunction of $(-\Delta+1)^{-1}\left(v_{0}^{2} \cdot\right)$ will from now on be denoted by $\varphi$. Additionally, we show that $\left(0, v_{0}, \underline{\mu}(\omega, n)\right)$ is a bifurcation point for (2) by proving that the positive minimizers of $\left.I\right|_{\mathcal{M}_{b}}$ converge to $\left(0, v_{0}\right)$ as $b$ tends to $\underline{\mu}(\omega, n)$ from the left.

Theorem 3. Let $n \in\{1,2,3\}, q=2$ and $\omega>1$.

(i) If $0 \leq b<\mu(\omega, n)$ then $\kappa_{b}>I\left(0, v_{0}\right)$ and $\kappa_{b}$ is attained at a positive solution of $\overline{(2)}$.

(ii) If $\underline{\mu}(\omega, n) \leq b<1$ then $\kappa_{b}=I\left(0, v_{0}\right)$ and $\kappa_{b}$ is not attained.

(iii) For every sequence $\left(b_{j}\right)$ converging to $\underline{\mu}(\omega, n)$ from the left and every sequence of positive minimizers of $\left.I\right|_{\mathcal{M}_{b_{j}}}$ we have $\left(u_{j}, v_{j}\right) \rightarrow\left(0, v_{0}\right)$ and $\frac{u_{j}}{\left\|u_{j}\right\|} \rightarrow \varphi$.

In Theorem 3 we excluded the case $\omega=1$ which is of different nature. The minimizers of $\left.I\right|_{\mathcal{M}_{b}}$ for $\omega=1$ can be determined explicitly and we refer the reader to Appendix A.

Remark 3. (i) Using different methods Ambrosetti and Colorado [1] and de Figueiredo and Lopes [5] proved the existence of positive solutions of (2) for $b<\underline{\mu}(\omega, n)$ as well. However, their papers do not contain any statement about the energy level of the constructed solutions. In particular it was not clear whether Sirakov's bound $\tilde{\mu}(\omega, n)$ was smaller than $\mu(\omega, n)$ or vice versa. Now we know $\tilde{\mu}(\omega, n) \leq \underline{\mu}(\omega, n)$ and that $\underline{\mu}(\omega, n)$ is sharp. Moreover let us add that in the three-dimensional case $\tilde{\mu}(\bar{\omega}, 3)$ vanishes as $\omega \rightarrow \infty$ whereas $\underline{\mu}(\omega, 3)$ stays away from zero. This observation is based 
on Birman-Schwinger bounds (cf. [6], p.6) for the number of eigenvalues of the Schrödinger operator $-\Delta+V$ below -1 where the Rollnik potential $V$ is given by $V=-\mu(\omega, 3) v_{0}^{2}$, see [5], p.151

(ii) It would be interesting to know if there are positive solutions of (2) for $b$ lying in $(\mu(\omega, n), b(\omega, 2, n))$ when $q=2$ or for $b$ in $(q-1, b(\omega, q, n))$ when $q>2$. For $\omega \approx 1$ and $b \neq q-1$ the existence of such solutions near $(1+b)^{-1 /(q-1)}\left(u_{0}, u_{0}\right)$ can be proved using the nondegeneracy of $u_{0}$ and the implicit function theorem. A variational approach proving the existence of a positive solution for a given $\omega \neq 1$ is missing, however.

Having submitted our paper we learned that the results of Chen and Zou [4] are almost identical to ours from Theorem 3, see Theorem 1.2 (ii), (iii) in [4]. Let us add that Remark 1(d) gives a partial answer to the question from remark $3.2(1)$ in [4].

\section{Preliminaries}

Throughout the paper we assume $n \in \mathbb{N}, 1<q<\frac{n}{(n-2)_{+}}$and $\omega \geq 1$. As in [11] the Sobolev norms $\|\cdot\|,\|\cdot\|_{\omega}$ are defined by

$$
\|u\|:=\left(\int_{\mathbb{R}^{n}}|\nabla u|^{2}+u^{2} d x\right)^{1 / 2}, \quad\|v\|_{\omega}^{2}:=\left(\int_{\mathbb{R}^{n}}|\nabla v|^{2}+\omega^{2} v^{2} d x\right)^{1 / 2}
$$

and $\|\cdot\|_{2},\|\cdot\|_{q},\|\cdot\|_{2 q}$ denote the standard norms on $L^{2}\left(\mathbb{R}^{n}\right), L^{q}\left(\mathbb{R}^{n}\right), L^{2 q}\left(\mathbb{R}^{n}\right)$, respectively. We will say that a function $(u, v)$ is nontrivial in case $(u, v) \neq$ $(0,0)$ and it is called fully nontrivial if we have $u \neq 0$ and $v \neq 0$. At several instances we will make use of the identity

$$
\left.I\right|_{\mathcal{N}_{b}}(u, v)=\frac{q-1}{2 q}\left(\|u\|^{2}+\|v\|_{\omega}^{2}\right) \quad \text { for all }(u, v) \in \mathcal{N}_{b}
$$

which in particular holds for all $(u, v) \in \mathcal{M}_{b}$.

The functions $u_{0}$ and $v_{0}:=\omega^{1 /(q-1)} u_{0}(\omega \cdot)$ having the property

$$
-\Delta u_{0}+u_{0}=u_{0}^{2 q-1} \text { in } \mathbb{R}^{n}, u_{0}>0, \quad-\Delta v_{0}+\omega^{2} v_{0}=v_{0}^{2 q-1} \text { in } \mathbb{R}^{n}, v_{0}>0
$$

will play a crucial role in the following analysis being the uniquely determined minimal energy solutions of the scalar equations associated to (2). As a consequence one has the inequalities

$$
\|u\| \geq \hat{c}_{0}\|u\|_{2 q},\|v\|_{\omega} \geq \omega^{\frac{2 q-n(q-1)}{2 q}} \hat{c}_{0}\|v\|_{2 q} \quad \text { for all } u, v \in H^{1}\left(\mathbb{R}^{n}\right)
$$

where $\hat{c}_{0}=\left\|u_{0}\right\|\left\|u_{0}\right\|_{2 q}^{-1}$. It is known that the equality cases occur if and only if $u, v$ are multiples of translates of $u_{0}, v_{0}$. Notice that the second inequality in (12) follows from the first by considering $u=v\left(\omega^{-1} \cdot\right)$. Moreover, we use

$$
I\left(u_{0}, 0\right)=\frac{q-1}{2 q}\left\|u_{0}\right\|^{2}=\frac{q-1}{2 q}\left\|u_{0}\right\|^{2} \cdot\left(\frac{\left\|u_{0}\right\|^{2}}{\left\|u_{0}\right\|_{2 q}^{2 q}}\right)^{\frac{1}{q-1}}=\frac{q-1}{2 q} \hat{c}_{0}^{\frac{2 q}{q-1}}
$$


as well as

$$
I\left(0, v_{0}\right)=\omega^{\frac{2 q-n(q-1)}{q-1}} I\left(u_{0}, 0\right), \quad\left\|u_{0}\right\|^{2}=\left\|u_{0}\right\|_{2 q}^{2 q}, \quad\left\|v_{0}\right\|_{\omega}^{2}=\left\|v_{0}\right\|_{2 q}^{2 q} .
$$

Let us now make a preliminary remark concerning the proof of Theorem 1 where the sharp criterion for the existence of a vector ground state is proved. From Theorem 2.1 in [11] we know that for all $b \geq 0$ a nonnegative minimizer of $\left.I\right|_{\mathcal{N}_{b}}$ exists which, by Lemma 3.3 in the same paper, is a ground state solution of (2). This ground state solution $(u, v)$ is either a vector ground state or it is semitrivial. In the latter case we must have $(u, v)=\left(u_{0}, 0\right)$ or $(u, v)=\left(0, v_{0}\right)$ so that the existence of a vector ground state is guaranteed if there is a function $(u, v) \in \mathcal{N}_{b}$ which satisfies $I(u, v)<\min \left\{I\left(u_{0}, 0\right), I\left(0, v_{0}\right)\right\}$. This idea has been worked out in [11], p.754. From (14) and $\omega \geq 1$ we infer that this is equivalent to

$$
I(u, v)<I\left(u_{0}, 0\right) \text { for some }(u, v) \in \mathcal{N}_{b} .
$$

We will use this criterion in the proof of Theorem 1 . Notice that in this case we automatically have $c_{b}<I\left(u_{0}, 0\right)$.

Moreover we will use the fact that in case $b \geq 0$ every ground state of $(2)$ is radially symmetric, radially nonincreasing and that it does not change sign in each component. Indeed, since $(u, v) \in \mathcal{N}_{b}$ is equivalent to $(|u|,|v|) \in \mathcal{N}_{b}$ and since every minimizer of $\left.I\right|_{\mathcal{N}_{b}}$ is a solution of (2) the strong minimum principle implies $|u|,|v|>0$ or $|u|>0, v=0$ or $u=0,|v|>0$ in $\mathbb{R}^{n}$. Hence, the symmetry results of Busca and Sirakov [3] and Ikoma [7] imply the radial symmetry and monotonicity of the functions $u, v$. Similarly, using Proposition 1 , one shows that minimizers of $\left.I\right|_{\mathcal{M}_{b}}$ are radially symmetric and do not change sign.

Finally we give the variational characterizations for $\bar{\mu}(\omega, n), \underline{\mu}(\omega, n)$. Since these numbers are the smallest characteristic values of the compact operators $\left(-\Delta+\omega^{2}\right)^{-1}\left(u_{0}^{2} \cdot\right),(-\Delta+1)^{-1}\left(v_{0}^{2} \cdot\right)$ we obtain from the Corant-Fischer minmax-principle

$$
\begin{aligned}
& \bar{\mu}(\omega, n)=\min \left\{\frac{\|v\|_{\omega}^{2}}{\left\|u_{0} v\right\|_{2}^{2}}: v \in H^{1}\left(\mathbb{R}^{n}\right), v \neq 0\right\}, \\
& \underline{\mu}(\omega, n)=\min \left\{\frac{\|u\|^{2}}{\left\|u v_{0}\right\|_{2}^{2}}: u \in H^{1}\left(\mathbb{R}^{n}\right), u \neq 0\right\},
\end{aligned}
$$

see also [1], p.454. Replacing $v$ by $v\left(\omega^{-1}\right.$.) in (16) we obtain the identity $\mu(\omega, n)=\bar{\mu}\left(\omega^{-1}, n\right)$ for all $\omega>0$ and in particular we get the inequality $\overline{\bar{\mu}}(\omega, n)>\bar{\mu}(1, n)=1=\mu(1, n)>\mu(\omega, n)$ for all $\omega>1$. Moreover, (16) and the estimates $\|\phi\|_{\omega}^{2} \geq \omega^{2}\|\bar{\phi}\|_{2}^{2}$ and $\left\|\bar{u}_{0} \phi\right\|_{2}^{2} \leq\left\|u_{0}\right\|_{\infty}^{2}\|\phi\|_{2}^{2}$ imply

$$
\bar{\mu}(\omega, n) \geq\left\|u_{0}\right\|_{\infty}^{-2} \omega^{2}
$$

which we claimed to hold in Remark 1 (d).

\section{Proof of Theorem 1}

Proof of Theorem 1, part (i): 
Let $b<b(\omega, q, n)$. We assume for contradiction that a vector ground state $(u, v) \in \mathcal{N}_{b}$ of $(2)$ exists. The unique continuation principle implies $u v \not \equiv 0$ so that the definition of $b(\omega, q, n)$ given in (5) gives the following inequality

$$
\frac{\hat{c}_{0}^{-2 q}\left(\|u\|^{2}+\|v\|_{\omega}^{2}\right)^{q}-\|u\|_{2 q}^{2 q}-\|v\|_{2 q}^{2 q}}{2\|u v\|_{q}^{q}}>b .
$$

Moreover $(u, v) \in \mathcal{N}_{b}$ implies $\|u\|^{2}+\|v\|_{\omega}^{2}=\|u\|_{2 q}^{2 q}+\|v\|_{2 q}^{2 q}+2 b\|u v\|_{q}^{q}$ and thus

$$
I(u, v) \stackrel{(11)}{=} \frac{q-1}{2 q}\left(\|u\|^{2}+\|v\|_{\omega}^{2}\right)=\frac{q-1}{2 q}\left(\frac{\left(\|u\|^{2}+\|v\|_{\omega}^{2}\right)^{q}}{\|u\|_{2 q}^{2 q}+\|v\|_{2 q}^{2 q}+2 b\|u v\|_{q}^{q}}\right)^{\frac{1}{q-1}}
$$

so that elementary rearrangements of $(19)$ and $\left(u_{0}, 0\right) \in \mathcal{N}_{b}$ yield

$$
I(u, v)>\frac{q-1}{2 q} \hat{c}_{0}^{\frac{2 q}{q-1}} \stackrel{(13)}{=} I\left(u_{0}, 0\right) \geq c_{b} .
$$

In particular $(u, v)$ is not a ground state which contradicts our assumption. Hence, every minimizer of $\left.I\right|_{\mathcal{N}_{b}}$ is semitrivial and we obtain $c_{b}=I\left(u_{0}, 0\right)$ for all $b<b(\omega, q, n)$.

Proof of Theorem 1, part (ii):

Now let $b>b(\omega, q, n)$. Then there are functions $u, v \in H^{1}\left(\mathbb{R}^{n}\right)$ satisfying $u v \neq 0$ and

$$
\frac{\hat{c}_{0}^{-2 q}\left(\|u\|^{2}+\|v\|_{\omega}^{2}\right)^{q}-\|u\|_{2 q}^{2 q}-\|v\|_{2 q}^{2 q}}{2\|u v\|_{q}^{q}}<b .
$$

Replacing $(u, v)$ by $(r u, r v)$ for

$$
r=\left(\frac{\|u\|^{2}+\|v\|_{\omega}^{2}}{\|u\|_{2 q}^{2 q}+\|v\|_{2 q}^{2 q}+2 b\|u v\|_{q}^{q}}\right)^{\frac{1}{2 q-2}}
$$

we may assume $(u, v) \in \mathcal{N}_{b}$. Then the same rearrangements as in the proof of part (i) give $I(u, v)<I\left(u_{0}, 0\right)$ so that the sufficient criterion (15) for the existence of a vector ground state with energy $c_{b}<I\left(u_{0}, 0\right)$ is satisfied. This proves the claim.

\section{Proof of Lemma 1}

Let $u, v \in H^{1}\left(\mathbb{R}^{n}\right)$ satisfy $u v \neq 0$ and set $r:=\|v\|_{2 q}\|u\|_{2 q}^{-1}$. From Hölder's inequality we get $\|u v\|_{q}^{q} \leq\|u\|_{2 q}^{q}\|v\|_{2 q}=r\|u\|_{2 q}^{2 q}$ so that (12) implies

$$
\frac{\hat{c}_{0}^{-2 q}\left(\|u\|^{2}+\|v\|_{\omega}^{2}\right)^{q}-\|u\|_{2 q}^{2 q}-\|v\|_{2 q}^{2 q}}{2\|u v\|_{q}^{q}} \geq \frac{\left(1+\omega^{\frac{2 q-n(q-1)}{q}} r^{2}\right)^{q}-1-r^{2 q}}{2 r^{q}} .
$$

Since this number is nonnegative we obtain $b(\omega, q, n) \geq 0$ so that claim (i) is proved. In case $q \geq 2$ we set $\hat{\omega}:=\omega^{(2 q-n(q-1)) /(2 q)} \geq 1$ and the estimate from 
part (ii) of the Lemma follows from

$$
\begin{aligned}
\frac{\hat{c}_{0}^{-2 q}\left(\|u\|^{2}+\|v\|_{\omega}^{2}\right)^{q}-\|u\|_{2 q}^{2 q}-\|v\|_{2 q}^{2 q}}{2\|u v\|_{q}^{q}} & \geq \inf _{r>0} \frac{\left(1+\hat{\omega}^{2} r^{2}\right)^{q}-1-r^{2 q}}{2 r^{q}} \\
& \geq \hat{\omega}^{q} \cdot \inf _{r>0} \frac{\left(1+(\hat{\omega} r)^{2}\right)^{q}-1-(\hat{\omega} r)^{2 q}}{2(\hat{\omega} r)^{q}} \\
& =\hat{\omega}^{q} \cdot \inf _{t>0} \frac{\left(1+t^{2}\right)^{q}-1-t^{2 q}}{2 t^{q}} \\
& =\omega^{\frac{2 q-n(q-1)}{2}}\left(2^{q-1}-1\right) .
\end{aligned}
$$

\section{Proof of Lemma 2}

Throughout the proof we will use the identities

$$
\left\|u_{0}\right\|_{2}^{2}=\frac{2 q-n(q-1)}{2 q}\left\|u_{0}\right\|^{2}, \quad\left\|\nabla u_{0}\right\|_{2}^{2}=\frac{n(q-1)}{2 q}\left\|u_{0}\right\|^{2}
$$

which are taken from $(4.13)-(4.16)$ in [11].

First we show $b(\omega, 2, n) \leq \bar{\mu}(\omega, n)$. In (5) we use the test function $\left(u_{0}, \alpha \phi\right)$ for $\alpha>0$ and $\phi \in H^{1}\left(\mathbb{R}^{n}\right), \phi \neq 0$. From $\left\|u_{0}\right\|^{2}=\left\|u_{0}\right\|_{4}^{4}$ and $\hat{c}_{0}=\left\|u_{0}\right\|\left\|u_{0}\right\|_{4}^{-1}$ we obtain

$$
\begin{aligned}
b(\omega, 2, n) & \leq \inf _{\alpha>0} \frac{\hat{c}_{0}^{-4}\left(\left\|u_{0}\right\|^{2}+\|\alpha \phi\|_{\omega}^{2}\right)^{2}-\left\|u_{0}\right\|_{4}^{4}-\|\alpha \phi\|_{4}^{4}}{2\left\|u_{0}(\alpha \phi)\right\|_{2}^{2}} \\
& =\inf _{\alpha>0} \frac{2 \hat{c}_{0}^{-4}\left\|u_{0}\right\|^{2}\|\phi\|_{\omega}^{2} \cdot \alpha^{2}+\alpha^{4}\left(\hat{c}_{0}^{-4}\|\phi\|_{\omega}^{4}-\|\phi\|_{4}^{4}\right)}{2 \alpha^{2}\left\|u_{0} \phi\right\|_{2}^{2}} \\
& =\frac{\|\phi\|_{\omega}^{2}}{\left\|u_{0} \phi\right\|_{2}^{2}}
\end{aligned}
$$

so that (16) yields $b(\omega, 2, n) \leq \bar{\mu}(\omega, n)$ since $\phi$ was arbitrary. Theorem 1.4 in [5] gives $\bar{\mu}(\omega, 1)=\frac{\omega(\omega+1)}{2}$ which proves the estimate for $n=1$. In order to prove the explicit bounds for $n \in\{2,3\}$ we take $\phi=u_{0}$ and use the identities (20) to get

$b(\omega, 2, n) \leq \bar{\mu}(\omega, n) \leq \frac{\left\|u_{0}\right\|_{\omega}^{2}}{\left\|u_{0}\right\|_{4}^{4}}=\frac{\left\|u_{0}\right\|_{\omega}^{2}}{\left\|u_{0}\right\|^{2}}=\frac{\left\|\nabla u_{0}\right\|_{2}^{2}}{\left\|u_{0}\right\|^{2}}+\omega^{2} \frac{\left\|u_{0}\right\|_{2}^{2}}{\left\|u_{0}\right\|^{2}}=\frac{4-n}{4} \omega^{2}+\frac{n}{4}$.

It therefore remains to prove part (i),(iii) of the Lemma and the estimate (6).

For $u, v \in H^{1}\left(\mathbb{R}^{n}\right)$ satisfying $u v \neq 0$ we consider the test functions $(u(k \cdot)$, $v(k \cdot))$ where $k$ is chosen in an optimal way. From $\|u(k \cdot)\|^{2}=k^{2-n}\|\nabla u\|_{2}^{2}+$ $k^{-n}\|u\|_{2}^{2}$ and $\|v(k \cdot)\|_{\omega}^{2}=k^{2-n}\|\nabla v\|_{2}^{2}+k^{-n} \omega^{2}\|v\|_{2}^{2}$ ee obtain

$$
\begin{aligned}
& b(\omega, q, n) \\
& =\inf _{k>0} \frac{k^{n(1-q)} \hat{c}_{0}^{-2 q}\left(\|u\|_{2}^{2}+\omega^{2}\|v\|_{2}^{2}+k^{2}\left(\|\nabla u\|_{2}^{2}+\|\nabla v\|_{2}^{2}\right)\right)^{q}-\|u\|_{2 q}^{2 q}-\|v\|_{2 q}^{2 q}}{2\|u v\|_{q}^{q}} \\
& =\frac{C_{n, q}\left(\|u\|_{2}^{2}+\omega^{2}\|v\|_{2}^{2}\right)^{\frac{2 q-n(q-1)}{2}}\left(\|\nabla u\|_{2}^{2}+\|\nabla v\|_{2}^{2}\right)^{\frac{n(q-1)}{2}}-\|u\|_{2 q}^{2 q}-\|v\|_{2 q}^{2 q}}{2\|u v\|_{q}^{q}}
\end{aligned}
$$


where $C_{n, q}$ is given by

$$
\begin{aligned}
C_{n, q} & =\left(\frac{n(q-1)}{2 q-n(q-1)}\right)^{\frac{n(1-q)}{2}}\left(\frac{2 q}{2 q-n(q-1)}\right)^{q} \hat{c}_{0}^{-2 q} \\
& =\left(\frac{2 q}{n(q-1)}\right)^{\frac{n(q-1)}{2}}\left(\frac{2 q}{2 q-n(q-1)}\right)^{\frac{2 q-n(q-1)}{2}} \hat{c}_{0}^{-2 q} \\
& =\left(\frac{\left\|u_{0}\right\|^{2}}{\left\|\nabla u_{0}\right\|_{2}^{2}}\right)^{\frac{n(q-1)}{2}}\left(\frac{\left\|u_{0}\right\|^{2}}{\left\|u_{0}\right\|_{2}^{2}}\right)^{\frac{2 q-n(q-1)}{2}}\left(\frac{\left\|u_{0}\right\|}{\left\|u_{0}\right\|_{2 q}}\right)^{-2 q} \\
& =\frac{\left\|u_{0}\right\|_{2 q}^{2 q}}{\left\|\nabla u_{0}\right\|_{2}^{n(q-1)}\left\|u_{0}\right\|_{2}^{2 q-n(q-1)}},
\end{aligned}
$$

see (20). Notice that the infimum over all positive $k$ is attained at

$$
k_{\text {min }}=\left(\frac{\|u\|_{2}^{2}+\omega^{2}\|v\|_{2}^{2}}{\|\nabla u\|_{2}^{2}+\|\nabla v\|_{2}^{2}} \cdot \frac{n(q-1)}{2 q-n(q-1)}\right)^{1 / 2}
$$

Now we choose $(u, v):=\left(u_{0}, \alpha u_{0}(\beta \cdot)\right)$ for $\alpha>0, \beta \geq 1$. Since $\beta \geq 1$ and $u_{0}$ is radially symmetric and radially decreasing we have $\left\|u_{0} u_{0}(\beta \cdot)\right\|_{q}^{q} \geq \| u_{0}$ $(\beta \cdot) u_{0}(\beta \cdot)\left\|_{q}^{q}=\beta^{-n}\right\| u_{0} \|_{2 q}^{2 q}$. This and the above estimate give

$$
b(\omega, q, n) \leq \frac{\left(1+\omega^{2} \alpha^{2} \beta^{-n}\right)^{\frac{2 q-n(q-1)}{2}}\left(1+\alpha^{2} \beta^{2-n}\right)^{\frac{n(q-1)}{2}}-1-\alpha^{2 q} \beta^{-n}}{2 \alpha^{q} \beta^{-n}} .
$$

Now it remains to pick special values for $\alpha>0, \beta \geq 1$. The assertion $b(\omega, q, n) \leq 0$ for $1<q<2$ results from the choice $(\alpha, \beta)=\left(\alpha_{k}, 1\right)$ where $\left(\alpha_{k}\right)$ is a positive null sequence. In case $q=2, n=3, \omega^{2}>5$ we choose $\beta=1$ and the corresponding optimal value for $\alpha$ is $\alpha=\frac{1}{\sqrt{2} \omega} \sqrt{-1+\omega^{2}-\sqrt{1+3 \omega^{2}}}$. This gives the estimate (6). The bounds for $q>2$ result from the choices $\alpha=\omega^{-1 / 2}, \beta=\omega$ in case $n=1$ and $\alpha=\beta=1$ in case $n \in\{2,3\}$.

\section{Proof of Lemma 3}

First we prove part (i), so let $b<0$. For all $(u, v) \in \mathcal{M}_{b}$ we have

$$
\|u\|^{2}=\|u\|_{2 q}^{2 q}+b\|u v\|_{q}^{q} \leq\|u\|_{2 q}^{2 q}, \quad\|v\|_{\omega}^{2}=\|v\|_{2 q}^{2 q}+b\|u v\|_{q}^{q} \leq\|v\|_{2 q}^{2 q} .
$$

This implies

$$
\begin{aligned}
I(u, v) & \stackrel{(11)}{=} \frac{q-1}{2 q}\left(\|u\|^{2}+\|v\|_{\omega}^{2}\right) \\
& \geq \frac{q-1}{2 q}\left(\|u\|^{2} \cdot\left(\frac{\|u\|^{2}}{\|u\|_{2 q}^{2 q}}\right)^{\frac{1}{q-1}}+\|v\|_{\omega}^{2} \cdot\left(\frac{\|v\|_{\omega}^{2}}{\|v\|_{2 q}^{2 q}}\right)^{\frac{1}{q-1}}\right) \\
& =\frac{q-1}{2 q}\left(\left(\frac{\|u\|}{\|u\|_{2 q}}\right)^{\frac{2 q}{q-1}}+\left(\frac{\|v\|_{\omega}}{\|v\|_{2 q}}\right)^{\frac{2 q}{q-1}}\right) \\
& \stackrel{(12)}{\geq} \frac{q-1}{2 q}\left(1+\omega^{\frac{2 q-n(q-1)}{q-1}}\right) \hat{c}_{0}^{\frac{2 q}{q-1}}
\end{aligned}
$$




$$
\begin{aligned}
& \stackrel{(13)}{=}\left(1+\omega^{\frac{2 q-n(q-1)}{q-1}}\right) I\left(u_{0}, 0\right) \\
& \stackrel{(14)}{=} I\left(u_{0}, 0\right)+I\left(0, v_{0}\right)
\end{aligned}
$$

where equality holds if and only if $u v \equiv 0$ and $u, v$ are multiples of translates of $u_{0}, v_{0}$, respectively. This being impossible we obtain

$$
I(u, v)>I\left(u_{0}, 0\right)+I\left(0, v_{0}\right) \quad \text { for all }(u, v) \in \mathcal{M}_{b} .
$$

The proof of (i) will be accomplished once we show $\kappa_{b} \leq I\left(u_{0}, 0\right)+I\left(0, v_{0}\right)$. To this end let $\chi \in C_{0}^{\infty}\left(\mathbb{R}^{n}\right)$ denote a cut-off function satisfying $\operatorname{supp}(\chi) \subset B_{1}$, $\left.\chi\right|_{B_{1 / 2}} \equiv 1$. We set $\tilde{u}_{k}(x):=u_{0}(x) \chi\left(\frac{x}{k}\right), \tilde{v}_{k}(x):=v_{0}(x) \chi\left(\frac{x}{k}\right)$ and

$$
\begin{aligned}
& u_{k}(x):=\left(\frac{\left\|\tilde{u}_{k}\right\|}{\left\|\tilde{u}_{k}\right\|_{2 q}^{q}}\right)^{\frac{1}{q-1}} \cdot \tilde{u}_{k}\left(x+2 k e_{1}\right), \\
& v_{k}(x):=\left(\frac{\left\|\tilde{v}_{k}\right\|_{\omega}}{\left\|\tilde{v}_{k}\right\|_{2 q}^{q}}\right)^{\frac{1}{q-1}} \cdot \tilde{v}_{k}\left(x-2 k e_{1}\right) .
\end{aligned}
$$

Then we have $\operatorname{supp}\left(u_{k}\right) \subset \overline{B_{k}\left(-2 k e_{1}\right)}$ and $\operatorname{supp}\left(v_{k}\right) \subset \overline{B_{k}\left(2 k e_{1}\right)}$ which implies $u_{k} v_{k} \equiv 0$. From $\left\|u_{k}\right\|^{2}=\left\|u_{k}\right\|_{2 q}^{2 q},\left\|v_{k}\right\|_{\omega}^{2}=\left\|v_{k}\right\|_{2 q}^{2 q}$ we infer $\left(u_{k}, v_{k}\right) \in \mathcal{M}_{b}$ for all $k \in \mathbb{N}$ and thus

$$
\begin{aligned}
\kappa_{b} & \leq\left.\lim _{k \rightarrow \infty} I\right|_{\mathcal{M}_{b}}\left(u_{k}, v_{k}\right) \\
& \stackrel{(11)}{=} \frac{q-1}{2 q} \lim _{k \rightarrow \infty}\left(\left\|u_{k}\right\|^{2}+\left\|v_{k}\right\|_{\omega}^{2}\right) \\
& =\frac{q-1}{2 q} \lim _{k \rightarrow \infty}\left(\left(\frac{\left\|\tilde{u}_{k}\right\|}{\left\|\tilde{u}_{k}\right\|_{2 q}}\right)^{\frac{2 q}{q-1}}+\left(\frac{\left\|\tilde{v}_{k}\right\|_{\omega}}{\left\|\tilde{v}_{k}\right\|_{2 q}}\right)^{\frac{2 q}{q-1}}\right) \\
& =\frac{q-1}{2 q}\left(\left(\frac{\left\|u_{0}\right\|}{\left\|u_{0}\right\|_{2 q}}\right)^{\frac{2 q}{q-1}}+\left(\frac{\left\|v_{0}\right\|_{\omega}}{\left\|v_{0}\right\|_{2 q}}\right)^{\frac{2 q}{q-1}}\right) \\
& =\frac{q-1}{2 q}\left(1+\omega^{\frac{2 q-n(q-1)}{q-1}}\right) \hat{c}_{0}^{\frac{2 q}{q-1}} \\
& =I\left(u_{0}, 0\right)+I\left(0, v_{0}\right)
\end{aligned}
$$

which proves part (i).

Now let us prove part (ii), so let $b>b(\omega, q, n)$. By Theorem 1 there is a vector ground state $(u, v)$ of $(2)$ so that $(u, v) \in \mathcal{M}_{b}$ and $I(u, v)=c_{b}$. From (9) we infer

$$
c_{b} \leq \kappa_{b} \leq I(u, v)=c_{b}
$$

which implies that $\kappa_{b}=c_{b}$ is attained at $(u, v)$. Vice versa, if $\kappa_{b}$ is attained at $(u, v) \in \mathcal{M}_{b}$ then $I(u, v)=\kappa_{b}$. From above we get $\kappa_{b}=c_{b}$ so that $I(u, v)=c_{b}$ implies that $(u, v)$ minimizes $\left.I\right|_{\mathcal{N}_{b}}$. Hence, $(u, v)$ is a vector ground state of (2) which is all we had to show. 


\section{Proof of Theorem 2}

First we show that minimizers of $\left.I\right|_{\mathcal{M}_{b}}$ are solutions of (2). The corresponding result for $q=2$ can be found in [14], Proposition 1.1. Our proof follows the same ideas.

Proposition 1. Let $n \in\{1,2,3\}, 2 \leq q<\frac{n}{(n-2)_{+}}, \omega \geq 1$ and $b \in[0, q-1)$. Then every minimizer of $\left.I\right|_{\mathcal{M}_{b}}$ is a solution of (2).

Proof. For $(u, v) \in \mathcal{M}_{b}$ we set $H_{1}(u, v):=\|u\|^{2}-\|u\|_{2 q}^{2 q}-b\|u v\|_{q}^{q}$ and $H_{2}(u, v)$ $:=\|v\|_{\omega}^{2}-\|v\|_{2 q}^{2 q}-b\|u v\|_{q}^{q}$ so that $b(q-2) \geq 0$ implies

$$
\begin{aligned}
H_{1}^{\prime}(u, v)[u, 0] & =2\|u\|^{2}-2 q\|u\|_{2 q}^{2 q}-q b\|u v\|_{q}^{q} \\
& =-2(q-1)\|u\|_{2 q}^{2 q}-(q-2) b\|u v\|_{q}^{q}<0, \\
H_{2}^{\prime}(u, v)[0, v] & =2\|v\|_{\omega}^{2}-2 q\|v\|_{2 q}^{2 q}-q b\|u v\|_{q}^{q} \\
& =-2(q-1)\|v\|_{2 q}^{2 q}-(q-2) b\|u v\|_{q}^{q}<0
\end{aligned}
$$

and thus $\operatorname{ran}\left(H_{1}^{\prime}(u, v), H_{2}^{\prime}(u, v)\right)=\mathbb{R}^{2}$. As in [14] the Lagrange multiplier rule gives real numbers $L_{1}, L_{2} \in \mathbb{R}$ such that

$$
I^{\prime}(u, v)+L_{1} H_{1}^{\prime}(u, v)+L_{2} H_{2}^{\prime}(u, v)=0 .
$$

Our aim is to show $L_{1}=L_{2}=0$ and we assume $\left(L_{1}, L_{2}\right) \neq(0,0)$ for contradiction. Testing $(21)$ with $(u, 0)$ and $(0, v)$ we obtain the linear system

$$
\begin{aligned}
& 0=\left((2-2 q)\|u\|_{2 q}^{2 q}+(2-q) b\|u v\|_{q}^{q}\right) L_{1}-q b\|u v\|_{q}^{q} L_{2}, \\
& 0=\left((2-2 q)\|v\|_{2 q}^{2 q}+(2-q) b\|u v\|_{q}^{q}\right) L_{2}-q b\|u v\|_{q}^{q} L_{1}
\end{aligned}
$$

where we used $I^{\prime}(u, v)[(u, 0)]=H_{1}(u, v)=0, I^{\prime}(u, v)[(0, v)]=H_{2}(u, v)=0$ and $(u, v) \in \mathcal{M}_{b}$. From $\left(L_{1}, L_{2}\right) \neq(0,0)$ we infer that the determinant vanishes and thus

$$
\begin{aligned}
0= & \left((2-2 q)\|u\|_{2 q}^{2 q}+(2-q) b\|u v\|_{q}^{q}\right) \cdot\left((2-2 q)\|v\|_{2 q}^{2 q}+(2-q) b\|u v\|_{q}^{q}\right) \\
& -\left(-q b\|u v\|_{q}^{q}\right)^{2} \\
= & 4(1-q)\left(\left(b\|u v\|_{q}^{q}\right)^{2}-\frac{q-2}{2} b\|u v\|_{q}^{q}\left(\|u\|_{2 q}^{2 q}+\|v\|_{2 q}^{2 q}\right)-(q-1)\|u\|_{2 q}^{2 q}\|v\|_{2 q}^{2 q}\right) .
\end{aligned}
$$

Solving this equation for $b\|u v\|_{q}^{q}$ gives

$$
\begin{aligned}
4 b\|u v\|_{q}^{q}= & (q-2)\left(\|u\|_{2 q}^{2 q}+\|v\|_{2 q}^{2 q}\right) \\
& +\sqrt{(q-2)^{2}\left(\|u\|_{2 q}^{2 q}+\|v\|_{2 q}^{2 q}\right)^{2}+16(q-1)\|u\|_{2 q}^{2 q}\|v\|_{2 q}^{2 q}} \\
\geq & 2(q-2)\|u\|_{2 q}^{q}\|v\|_{2 q}^{q} \\
& +\sqrt{4(q-2)^{2}\|u\|_{2 q}^{2 q}\|v\|_{2 q}^{2 q}+16(q-1)\|u\|_{2 q}^{2 q}\|v\|_{2 q}^{2 q}} \\
= & 4(q-1)\|u\|_{2 q}^{q}\|v\|_{2 q}^{q} \\
\geq & 4(q-1)\|u v\|_{q}^{q}
\end{aligned}
$$


which contradicts $b<q-1$. Notice that in case $u v \equiv 0$ the last inequality is strict. Hence, $L_{1}=L_{2}=0$ which is all we had to show.

We rewrite the minimization problem inf $\left.I\right|_{\mathcal{M}_{b}}$ with the aid of a fibering map. For all $u, v \in H^{1}\left(\mathbb{R}^{n}\right)$ satisfying $u, v \neq 0$ we define $\beta_{u, v}: \mathbb{R}_{\geq 0} \times \mathbb{R}_{\geq 0} \rightarrow \mathbb{R}$ by

$$
\beta_{u, v}(s, t):=I(\sqrt{s} u, \sqrt{t} v)
$$

which implies

$$
\begin{aligned}
& \partial_{s} \beta_{u, v}(s, t)=(2 s)^{-1}\left(\|\sqrt{s} u\|^{2}-\|\sqrt{s} u\|_{2 q}^{2 q}-b\|(\sqrt{s} u)(\sqrt{t} v)\|_{q}^{q}\right) \\
& \partial_{t} \beta_{u, v}(s, t)=(2 t)^{-1}\left(\|\sqrt{t} v\|_{\omega}^{2}-\|\sqrt{t} v\|_{2 q}^{2 q}-b\|(\sqrt{s} u)(\sqrt{t} v)\|_{q}^{q}\right)
\end{aligned}
$$

for all $s, t>0$ and in particular $(\sqrt{s} u, \sqrt{t} v) \in \mathcal{M}_{b}$ if and only if $(s, t)$ is a critical point of $\beta_{u, v}$. In the following Proposition we prove that $\beta_{u, v}$ has exactly one critical point.

Proposition 2. Let $n \in\{1,2,3\}, 2<q<\frac{n}{(n-2)_{+}}, \omega>1, b \in[0, q-1)$ and let $u, v \in H^{1}\left(\mathbb{R}^{n}\right)$ satisfy $u, v \neq 0$. Then $\beta_{u, v}$ has precisely one critical point $(s(u, v), t(u, v))$ in $\mathbb{R}_{>0} \times \mathbb{R}_{>0}$ and the critical value is a strict global maximum over $\mathbb{R}_{\geq 0} \times \mathbb{R}_{\geq 0}$. In particular $(\sqrt{s} u, \sqrt{t} v) \in \mathcal{M}_{b}$ if and only if $(s, t)=$ $(s(u, v), t(u, \bar{v}))$.

Proof. Let $u, v \in H^{1}\left(\mathbb{R}^{n}\right)$ and $u, v \neq 0$. From

$$
\beta_{u, v}(s, t)=\frac{1}{2}\left(s\|u\|^{2}+t\|v\|_{\omega}^{2}\right)-\frac{1}{2 q}\left(s^{q}\|u\|_{2 q}^{2 q}+t^{q}\|v\|_{2 q}^{2 q}+2 s^{\frac{q}{2}} t^{\frac{q}{2}} b\|u v\|_{q}^{q}\right)
$$

we infer that $\beta:=\beta_{u, v}$ is continuous on $\mathbb{R}_{\geq 0} \times \mathbb{R}_{\geq 0}$ and that $-\beta$ is coercive. This and $\partial_{s} \beta(0, t), \partial_{t} \beta(s, 0)>0$ for all $s, t \geq 0$ implies that $\beta$ attains a global maximum at some point $(\bar{s}, \bar{t})$ with $\bar{s}, \bar{t}>0$. It remains to show that the maximum is strict and that $\beta$ has no other critical points in $\mathbb{R}_{>0} \times \mathbb{R}_{>0}$.

In $\mathbb{R}_{>0} \times \mathbb{R}_{>0}$ the map $\beta$ is twice continuously differentiable and satisfies

$$
\begin{aligned}
& \partial_{s s} \beta(s, t)=-\frac{q-1}{2} s^{q-2}\|u\|_{2 q}^{2 q}-\frac{b(q-2)}{4} t^{\frac{q}{2}} s^{\frac{q-4}{2}}\|u v\|_{q}^{q}, \\
& \partial_{t t} \beta(s, t)=-\frac{q-1}{2} t^{q-2}\|v\|_{2 q}^{2 q}-\frac{b(q-2)}{4} s^{\frac{q}{2}} t^{\frac{q-4}{2}}\|u v\|_{q}^{q} \\
& \partial_{s t} \beta(s, t)=-\frac{q}{4}(s t)^{\frac{q-2}{2}} b\|u v\|_{q}^{q} .
\end{aligned}
$$


From $b(q-2) \geq 0$ we infer $\partial_{s s} \bar{\beta}(s, t), \partial_{t t} \bar{\beta}(s, t)<0$ as well as

$$
\begin{aligned}
4 \operatorname{det}\left(D^{2} \beta(s, t)\right)= & 4 \cdot\left(\partial_{s s} \beta(s, t) \cdot \partial_{t t} \beta(s, t)-\left(\partial_{s t} \beta(s, t)\right)^{2}\right) \\
= & -(q-1)(s t)^{q-2}\left(\left(b\|u v\|_{q}^{q}\right)^{2}-(q-1)\|u\|_{2 q}^{2 q}\|v\|_{2 q}^{2 q}\right. \\
& \left.-\frac{q-2}{2}\left(\left(\frac{t}{s}\right)^{\frac{q}{2}}\|v\|_{2 q}^{2 q}+\left(\frac{s}{t}\right)^{\frac{q}{2}}\|u\|_{2 q}^{2 q}\right) b\|u v\|_{q}^{q}\right) \\
\geq & -(q-1)(s t)^{q-2}\left(\left(b\|u v\|_{q}^{q}\right)^{2}-(q-1)\|u\|_{2 q}^{2 q}\|v\|_{2 q}^{2 q}\right. \\
& \left.-(q-2)\|u\|_{2 q}^{q}\|v\|_{2 q}^{q} b\|u v\|_{q}^{q}\right) \\
= & -(q-1)(s t)^{q-2}\left(b\|u v\|_{q}^{q}-(q-1)\|u\|_{2 q}^{q}\|v\|_{2 q}^{q}\right) . \\
& \left(b\|u v\|_{q}^{q}+\|u\|_{2 q}^{q}\|v\|_{2 q}^{q}\right)>0
\end{aligned}
$$

where the last inequality follows from Hölder's inequality and $b \in[0, q-1)$. Hence the Hessian of $\beta$ is negative definite in $\mathbb{R}_{>0} \times \mathbb{R}_{>0}$ which proves that the maximum is strict and that $(\bar{s}, \bar{t})$ is the only critical point of $\beta$.

As a consequence, for any given $u, v \in H^{1}\left(\mathbb{R}^{n}\right)$ with $u, v \neq 0$ we have

$$
\begin{aligned}
& I(\sqrt{s(u, v)} u, \sqrt{t(u, v)} v) \\
& =\max _{s, t>0} I(\sqrt{s} u, \sqrt{t} v) \\
& =\max _{\alpha>0} \max _{r>0} I(\sqrt{r} u, \sqrt{r} \alpha v) \\
& =\frac{q-1}{2 q} \max _{\alpha>0}\left(\frac{\left(\|u\|^{2}+\alpha^{2}\|v\|_{\omega}^{2}\right)^{q}}{\|u\|_{2 q}^{2 q}+\alpha^{2 q}\|v\|_{2 q}^{2 q}+2 b \alpha^{q}\|u v\|_{q}^{q}}\right)^{\frac{1}{q-1}}
\end{aligned}
$$

where the last identity result from the explicit computation of the maximizer. For notational convenience we set

$$
\hat{J}(u, v)=\max _{\alpha>0} J(u, \alpha v)
$$

where $J$ is given by

$$
J(u, v)=\frac{q-1}{2 q}\left(\frac{\left(\|u\|^{2}+\|v\|_{\omega}^{2}\right)^{q}}{\|u\|_{2 q}^{2 q}+\|v\|_{2 q}^{2 q}+2 b\|u v\|_{q}^{q}}\right)^{\frac{1}{q-1}}
$$

Now the proof of Theorem 2 is basically reduced to finding a nonnegative fully nontrivial minimizer of $\hat{J}$.

\section{Proof of Theorem 2:}

Let us first prove the estimate $\kappa_{b}>I\left(0, v_{0}\right)$. Since we will prove below that $\kappa_{b}$ is attained at a positive solution $(u, v) \in \mathcal{M}_{b}$ of (2) it is sufficient to prove the inequality $I(u, v)>I\left(0, v_{0}\right)$ for all $(u, v) \in \mathcal{M}_{b}$. So let $(u, v) \in \mathcal{M}_{b}$. Proposition 2 implies $s(u, v)=t(u, v)=1$ and (24) implies that the value $I(u, v)$ is given by $\max _{\alpha>0} J(u, \alpha v)$ and the maximum is strict. Hence we 
obtain

$$
\begin{aligned}
I(u, v) & >\max \left\{\lim _{\alpha \rightarrow 0} J(u, \alpha v), \lim _{\alpha \rightarrow \infty} J(u, \alpha v)\right\} \\
& =\frac{q-1}{2 q} \max \left\{\frac{\|u\|^{2 q}}{\|u\|_{2 q}^{2 q}}, \frac{\|v\|_{\omega}^{2 q}}{\|v\|_{2 q}^{2 q}}\right\}^{\frac{1}{q-1}}
\end{aligned}
$$

so that $\omega \geq 1$ implies

$$
I(u, v) \stackrel{(12)}{>} \frac{q-1}{2 q} \cdot \omega^{\frac{2 q-n(q-1)}{q-1}} \hat{c}_{0}^{\frac{2 q}{q-1}} \stackrel{(13),(14)}{=} I\left(0, v_{0}\right) .
$$

Next we show that $\hat{J}$ has a nonnegative fully nontrivial minimizer. The functional $\hat{J}$ satisfies $\hat{J}(\lambda u, \mu v)=\hat{J}(u, v)$ for all $\lambda, \mu \neq 0$ and $\hat{J}\left(|u|^{*},|v|^{*}\right) \leq$ $\hat{J}(u, v)$ where the ${ }^{*}$-operator denotes the Schwarz symmetrization (i.e. the spherical rearrangement) of the given function. For this reason there is a minimizing sequence $\left(u_{j}, v_{j}\right)$ of $\hat{J}$ consisting of Schwarz symmetric functions that satisfy $\left\|u_{j}\right\|_{2 q}=\left\|v_{j}\right\|_{2 q}=1$. Then (26) implies that $\left(u_{j}, v_{j}\right)$ is bounded in $H^{1}\left(\mathbb{R}^{n}\right) \times H^{1}\left(\mathbb{R}^{n}\right)$. Hence, there is a function $(\tilde{u}, \tilde{v}) \in H^{1}\left(\mathbb{R}^{n}\right) \times H^{1}\left(\mathbb{R}^{n}\right)$ and a subsequence of $\left(u_{j}, v_{j}\right)$, again denoted by $\left(u_{j}, v_{j}\right)$, which satisfies $\left(u_{j}, v_{j}\right) \rightarrow$ $(\tilde{u}, \tilde{v}),\left(u_{j}, v_{j}\right) \rightarrow(\tilde{u}, \tilde{v})$ in $L^{2 q}\left(\mathbb{R}^{n}\right) \times L^{2 q}\left(\mathbb{R}^{n}\right)$ and pointwise almost everywhere. This implies $\tilde{u}, \tilde{v} \geq 0,\|\tilde{u}\|_{2 q}=\|\tilde{v}\|_{2 q}=1$ and that $(\tilde{u}, \tilde{v})$ is a minimizer of $\hat{J}$. Indeed, we have

$$
J(\tilde{u}, \alpha \tilde{v}) \leq \liminf _{j \rightarrow \infty} J\left(\tilde{u}_{j}, \alpha \tilde{v}_{j}\right) \leq \liminf _{j \rightarrow \infty} \hat{J}\left(\tilde{u}_{j}, \tilde{v}_{j}\right) \quad \text { for all } \alpha>0
$$

and this implies

$$
\inf \hat{J}=\liminf _{j \rightarrow \infty} \hat{J}\left(u_{j}, v_{j}\right) \geq \max _{\alpha>0} J(\tilde{u}, \alpha \tilde{v})=\hat{J}(\tilde{u}, \tilde{v}) .
$$

From this we infer that $(u, v):=(\sqrt{s(\tilde{u}, \tilde{v})} \tilde{u}, \sqrt{t(\tilde{u}, \tilde{v})} \tilde{v}) \in \mathcal{M}_{b}$ is a minimizer of $\left.I\right|_{\mathcal{M}_{b}}$ since (24) and (25) give the following inequality for all $(\bar{u}, \bar{v}) \in \mathcal{M}_{b}$

$$
\begin{aligned}
I(\bar{u}, \bar{v}) & =I(\sqrt{s(\bar{u}, \bar{v})} \bar{u}, \sqrt{t(\bar{u}, \bar{v})} \bar{v}) \\
& =\hat{J}(\bar{u}, \bar{v}) \\
& \geq \hat{J}(\tilde{u}, \tilde{v}) \\
& =I(\sqrt{s(\tilde{u}, \tilde{v})} \tilde{u}, \sqrt{t(\tilde{u}, \tilde{v})} \tilde{v}) \\
& =I(u, v) .
\end{aligned}
$$

Now Lemma 1 implies that $(u, v)$ is a nonnegative fully nontrival solution of $(2)$ which, by the strong minimum principle, is positive.

\section{Proof of Theorem 3}

In the following let the function $\beta_{u, v}$ be given by formula (22) for $q=2$. As in the proof of Theorem 2 we first collect some properties of the fibering map. 
Proposition 3. Let $n \in\{1,2,3\}, \omega>1, b \in[0,1)$ and let $u, v \in H^{1}\left(\mathbb{R}^{n}\right)$ satisfy $u, v \neq 0$. Then $\beta_{u, v}$ has a critical point in $\mathbb{R}_{>0} \times \mathbb{R}_{>0}$ if and only if

$$
\frac{b\|u v\|_{2}^{2}}{\|u\|_{4}^{2}\|v\|_{4}^{2}}<\min \left\{\frac{\|u\|^{2}\|v\|_{4}^{2}}{\|u\|_{4}^{2}\|v\|_{\omega}^{2}}, \frac{\|u\|_{4}^{2}\|v\|_{\omega}^{2}}{\|u\|^{2}\|v\|_{4}^{2}}\right\} .
$$

In this case the critical point is unique and the critical value is a strict global maximum over $\mathbb{R}_{\geq 0} \times \mathbb{R}_{\geq 0}$. The maximizer $(s(u, v), t(u, v))$ is given by

$$
\begin{aligned}
& s(u, v)=\frac{\|v\|_{4}^{4}\|u\|^{2}-b\|u v\|_{2}^{2}\|v\|_{\omega}^{2}}{\|u\|_{4}^{4}\|v\|_{4}^{4}-b^{2}\|u v\|_{2}^{4}}, \\
& t(u, v)=\frac{\|u\|_{4}^{4}\|v\|_{\omega}^{2}-b\|u v\|_{2}^{2}\|u\|^{2}}{\|u\|_{4}^{4}\|v\|_{4}^{4}-b^{2}\|u v\|_{2}^{4}} .
\end{aligned}
$$

In particular we have $(\sqrt{s} u, \sqrt{t} v) \in \mathcal{M}_{b}$ if and only if $(s, t)=(s(u, v), t(u, v))$. Proof. From (23) we infer that $(s, t) \in \mathbb{R}_{>0} \times \mathbb{R}_{>0}$ is a critical point $\beta_{u, v}$ if and only if

$$
\|u\|^{2}=s\|u\|_{4}^{4}+t b\|u v\|_{2}^{2}, \quad\|v\|_{\omega}^{2}=t\|v\|_{4}^{4}+s b\|u v\|_{2}^{2} .
$$

Due to $b\|u v\|_{2}^{2} \leq b\|u\|_{4}^{2}\|v\|_{4}^{2}<\|u\|_{4}^{2}\|v\|_{4}^{2}$ this linear system has a unique solution which, formally, is given by (29). From $s(u, v), t(u, v)>0$ we get that condition (28) is necessary and sufficient for the existence of a critical point in $\mathbb{R}_{>0} \times \mathbb{R}_{>0}$. As in the proof of Proposition 2 one finds that in this case the Hessian of $\beta_{u, v}$ is negative definite in $\mathbb{R}_{>0} \times \mathbb{R}_{>0}$ and $\bar{\beta}_{s}(0, t), \bar{\beta}_{t}(s, 0)>0$ for all $s, t \geq 0$ so that the critical value associated to $(s(u, v), t(u, v))$ is a strict global maximum over $\mathbb{R}_{\geq 0} \times \mathbb{R}_{\geq 0}$.

As in (24) we obtain $I(\sqrt{s(u, v)} u, \sqrt{t(u, v)} v)=\max _{\alpha>0} J(u, \alpha v)$ whenever $(u, v)$ satisfies $(28)$. When (28) is not satisfied, however, the supremum of the function $\alpha \rightarrow J(u, \alpha v)$ over $(0, \infty)$ need not be attained in $(0, \infty)$. Nevertheless it will turn out to be useful to define

$$
\hat{J}(u, v):=\sup _{\alpha>0} J(u, \alpha v)=\frac{1}{4} \sup _{\alpha>0} \frac{\left(\|u\|^{2}+\alpha^{2}\|v\|_{\omega}^{2}\right)^{2}}{\|u\|_{4}^{4}+\alpha^{4}\|v\|_{4}^{4}+2 \alpha^{2} b\|u v\|_{2}^{2}} .
$$

In the proof of Theorem 3 we will need the following result.

Proposition 4. Let $n \in\{1,2,3\}, \omega>1$. Then $b \mapsto \kappa_{b}$ is continuous from the left on $[0,1)$.

Proof. Let $\left(b_{j}\right)$ be a sequence converging to $b \in(0,1)$ from the left. Continuity of translation in $L^{4}\left(\mathbb{R}^{n}\right)$ implies that for all $(u, v) \in \mathcal{M}_{b_{j}}$ there is a vector $z_{j} \in \mathbb{R}^{n}$ such that $b_{j}\|u v\|_{2}^{2}=b\left\|u\left(\cdot+z_{j}\right) v\right\|_{2}^{2}$, hence $\left(u\left(\cdot+z_{j}\right), v\right) \in \mathcal{M}_{b}$ and in particular

$$
\begin{aligned}
\left.I\right|_{\mathcal{M}_{b_{j}}}(u, v) & \stackrel{(11)}{=} \frac{1}{4}\left(\|u\|^{2}+\|v\|_{\omega}^{2}\right) \\
& =\frac{1}{4}\left(\left\|u\left(\cdot+z_{j}\right)\right\|^{2}+\|v\|_{\omega}^{2}\right) \\
& \left.\stackrel{(11)}{=} I\right|_{\mathcal{M}_{b}}\left(u\left(\cdot+z_{j}\right), v\right) \\
& \geq \kappa_{b} .
\end{aligned}
$$


Since $(u, v) \in \mathcal{M}_{b_{j}}$ and $b_{j}<b$ were chosen arbitrarily we obtain $\kappa_{b_{j}} \geq \kappa_{b}$ for all $j \in \mathbb{N}$ and thus $\liminf _{j \rightarrow \infty} \kappa_{b_{j}} \geq \kappa_{b}$.

Now let $(u, v) \in \mathcal{M}_{b}$ be arbitrary. Then $(u, v)$ satisfies condition (28) and for sufficiently large $j(28)$ is also satisfied for $b$ replaced by $b_{j}$. This implies $\left(\sqrt{s_{b_{j}}(u, v)} u, \sqrt{t_{b_{j}}(u, v)} v\right) \in \mathcal{M}_{b_{j}}$ where $s_{b_{j}}(u, v), t_{b_{j}}(u, v)$ are given by (29) for $b$ replaced by $b_{j}$. From $(u, v) \in \mathcal{M}_{b}$ and (29) we infer $s_{b_{j}}(u, v), t_{b_{j}}(u, v) \rightarrow 1$ as $j \rightarrow \infty$ and thus

$$
\begin{aligned}
\limsup _{j \rightarrow \infty} \kappa_{b_{j}} & \leq\left.\limsup _{j \rightarrow \infty} I\right|_{\mathcal{M}_{b_{j}}}\left(\sqrt{s_{b_{j}}(u, v)} u, \sqrt{t_{b_{j}}(u, v) v}\right) \\
& \stackrel{(11)}{=} \limsup _{j \rightarrow \infty} \frac{1}{4}\left(s_{b_{j}}(u, v)\|u\|^{2}+t_{b_{j}}(u, v)\|v\|_{\omega}^{2}\right) \\
& =\frac{1}{4}\left(\|u\|^{2}+\|v\|_{\omega}^{2}\right) \\
& \left.\stackrel{(11)}{=} I\right|_{\mathcal{M}_{b}}(u, v)
\end{aligned}
$$

and thus $\lim \sup _{j \rightarrow \infty} \kappa_{b_{j}} \leq \kappa_{b}$ since $(u, v) \in \mathcal{M}_{b}$ was arbitrary. This proves the claim.

In contrast to the case $q>2$ we find that for $b>0$ the closure of $\mathcal{M}_{b}$ in $H^{1}\left(\mathbb{R}^{n}\right) \times H^{1}\left(\mathbb{R}^{n}\right)$ may contain semitrivial elements when $q=2$. In the next Proposition we prove that the particular semitrivial element $\left(0, v_{0}\right)$ lies in closure of $\mathcal{M}_{b}$ precisely for $b \geq \underline{\mu}(\omega, n)$.

Proposition 5. Let $n \in\{1,2,3\}, \omega>1, b \geq 0$. Then we have $\left(0, v_{0}\right) \in \overline{\mathcal{M}_{b}}$ if and only if $b \geq \underline{\mu}(\omega, n)$.

Proof. First we assume $b \geq \mu(\omega, n)$, let $\varphi$ be the normalized positve eigenfunction of the operator $\left(-\Delta+\bar{\omega}^{2}\right)^{-1}\left(u_{0}^{2} \cdot\right)$ associated to the eigenvalue $\underline{\mu}(\omega, n)^{-1}$. By the variational characterization of $\underline{\mu}(\omega, n)$ given by $(17)$ and by $\bar{c}$ continuity of translation in $L^{4}\left(\mathbb{R}^{n}\right)$ there is a sequence $\left(z_{j}\right)$ in $\mathbb{R}^{n}$ such that the functions $u_{j}:=\varphi\left(\cdot-z_{j}\right)$ satisfy

$$
\frac{\left\|u_{j}\right\|^{2}}{\left\|u_{j} v_{0}\right\|_{4}^{2}}>b \quad \text { for } j \in \mathbb{N} \quad \text { and } \quad \frac{\left\|u_{j}\right\|^{2}}{\left\|u_{j} v_{0}\right\|_{4}^{2}} \rightarrow b \quad \text { as } j \rightarrow \infty .
$$

Then Hölder's inequality and $\left\|v_{0}\right\|<\left\|v_{0}\right\|_{\omega},\left\|v_{0}\right\|_{\omega}^{2}=\left\|v_{0}\right\|_{4}^{4}$ imply

$$
\frac{\left\|u_{j}\right\|^{2}}{\left\|u_{j}\right\|_{4}^{2}}=\frac{\|\varphi\|^{2}}{\|\varphi\|_{4}^{2}} \cdot \frac{\left\|v_{0}\right\|_{4}^{2}}{\left\|v_{0}\right\|_{4}^{2}} \leq \frac{\|\varphi\|^{2}}{\left\|\varphi v_{0}\right\|_{2}^{2}} \cdot\left\|v_{0}\right\|_{4}^{2} \stackrel{(17)}{\leq} \frac{\left\|v_{0}\right\|^{2}}{\left\|v_{0} v_{0}\right\|_{2}^{2}} \cdot\left\|v_{0}\right\|_{4}^{2}<\frac{\left\|v_{0}\right\|_{\omega}^{2}}{\left\|v_{0}\right\|_{4}^{2}}
$$

and thus

$$
\frac{b\left\|u_{j} v_{0}\right\|_{2}^{2}}{\left\|u_{j}\right\|_{4}^{2}\left\|v_{0}\right\|_{4}^{2}} \stackrel{(31)}{<} \frac{\left\|u_{j}\right\|^{2}}{\left\|u_{j}\right\|_{4}^{2}\left\|v_{0}\right\|_{4}^{2}} \stackrel{(32)}{=} \min \left\{\frac{\left\|u_{j}\right\|^{2}\left\|v_{0}\right\|_{4}^{2}}{\left\|u_{j}\right\|_{4}^{2}\left\|v_{0}\right\|_{\omega}^{2}}, \frac{\left\|u_{j}\right\|_{4}^{2}\left\|v_{0}\right\|_{\omega}^{2}}{\left\|u_{j}\right\|^{2}\left\|v_{0}\right\|_{4}^{2}}\right\} .
$$

Therefore $\left(u_{j}, v_{0}\right)$ satisfies (28) which, according to Proposition 3, implies $\left(\sqrt{s\left(u_{j}, v_{0}\right)} u_{j}, \sqrt{t\left(u_{j}, v_{0}\right)} v_{0}\right) \in \mathcal{M}_{b}$. The explicit formulas for $s\left(u_{j}, v_{0}\right)$, $t\left(u_{j}, v_{0}\right)$ from $(29)$ and $(31)$ give $s\left(u_{j}, v_{0}\right) \rightarrow 0, t\left(u_{j}, v_{0}\right) \rightarrow 1$ and hence

$$
\left(\sqrt{s\left(u_{j}, v_{0}\right)} u_{j}, \sqrt{t\left(u_{j}, v_{0}\right)} v_{0}\right) \rightarrow\left(0, v_{0}\right) \quad \text { as } j \rightarrow \infty .
$$


This implies $\left(0, v_{0}\right) \in \overline{\mathcal{M}_{b}}$.

Now assume $\left(0, v_{0}\right) \in \overline{\mathcal{M}_{b}}$ and let $\left(u_{j}, v_{j}\right)$ be a sequence in $\mathcal{M}_{b}$ that satisfies $\left(u_{j}, v_{j}\right) \rightarrow\left(0, v_{0}\right)$. Then the sequence $\phi_{j}:=u_{j}\left\|u_{j}\right\|_{4}^{-1}$ is bounded in $H^{1}\left(\mathbb{R}^{n}\right)$ because of

$$
\begin{aligned}
\left\|\phi_{j}\right\|^{2} & =\frac{\left\|u_{j}\right\|_{4}^{4}+b\left\|u_{j} v_{j}\right\|_{2}^{2}}{\left\|u_{j}\right\|_{4}^{2}} \\
& =\left\|u_{j}\right\|_{4}^{2}+b\left\|\phi_{j} v_{j}\right\|_{2}^{2} \\
& \leq b\left\|\phi_{j}\right\|_{4}^{2}\left\|v_{j}\right\|_{4}^{2}+o(1) \\
& =b\left\|v_{0}\right\|_{4}^{2}+o(1) \quad(j \rightarrow \infty) .
\end{aligned}
$$

Hence, we may take a subsequence $\left(\phi_{j}\right)$ that converges weakly and in $L_{l o c}^{4}\left(\mathbb{R}^{n}\right)$ to some function $\phi \in H^{1}\left(\mathbb{R}^{n}\right)$. From $\left(u_{j}, v_{j}\right) \rightarrow\left(0, v_{0}\right)$ and $\phi_{j} \rightarrow \phi$ in $L_{l o c}^{4}\left(\mathbb{R}^{n}\right)$ we obtain

$$
\left\|\phi_{j}\right\|^{2}=\left\|u_{j}\right\|_{4}^{2}+b\left\|\phi_{j} v_{j}\right\|_{2}^{2}=b\left\|\phi_{j} v_{0}\right\|_{2}^{2}+o(1)=b\left\|\phi v_{0}\right\|_{2}^{2}+o(1)
$$

as $j \rightarrow \infty$. By Sobolev's embedding theorem we moreover have $\left\|\phi_{j}\right\|=$ $\left\|u_{j}\right\|\left\|u_{j}\right\|_{4}^{-1} \geq c$ for some positive number $c$ so that (33) gives $\phi \neq 0$. This implies

$$
b \stackrel{(33)}{=} \liminf _{j \rightarrow \infty} \frac{\left\|\phi_{j}\right\|^{2}}{\left\|\phi v_{0}\right\|_{2}^{2}} \geq \frac{\|\phi\|^{2}}{\left\|\phi v_{0}\right\|_{2}^{2}} \stackrel{(17)}{\geq} \underline{\mu}(\omega, n) .
$$

Proof of Theorem 3, part (i):

The same reasoning as in the proof of Theorem 2 shows that the functional $\hat{J}$ defined in (30) attains its infimum over the set of all functions $u, v \in$ $H^{1}\left(\mathbb{R}^{n}\right)$ satisfying $u, v \neq 0$. We denote the minimizer by $(\tilde{u}, \tilde{v})$. In view of Proposition 3 we have to show that $(\tilde{u}, \tilde{v})$ satisfies condition (28) provided $0 \leq b<\mu(\omega, n)$. Once we have shown this we obtain as in the proof of Theorem 2 that $(u, v):=(s(\tilde{u}, \tilde{v}) \tilde{u}, t(\tilde{u}, \tilde{v}) \tilde{v})$ is a nonnegative minimizer of $\left.I\right|_{\mathcal{M}_{b}}$ and hence a positive solution of $(2)$.

So let $0 \leq b<\mu(\omega, n)$ and we assume for contradiction that $(\tilde{u}, \tilde{v})$ does not satisfy (28). Then we have $\frac{\|\tilde{v}\|_{\omega}}{\|\tilde{v}\|_{4}} \neq \frac{\|\tilde{u}\|}{\|\tilde{u}\|_{4}}$, for otherwise Hölder's inequality would imply

$$
b \geq \frac{b\|\tilde{u} \tilde{v}\|_{2}^{2}}{\|\tilde{u}\|_{4}^{2}\|\tilde{v}\|_{4}^{2}} \geq \min \left\{\frac{\|\tilde{u}\|^{2}\|\tilde{v}\|_{4}^{2}}{\|\tilde{u}\|_{4}^{2}\|\tilde{v}\|_{\omega}^{2}}, \frac{\|\tilde{u}\|_{4}^{2}\|\tilde{v}\|_{\omega}^{2}}{\|\tilde{u}\|^{2}\|\tilde{v}\|_{4}^{2}}\right\}=1
$$

which contradicts our assumption $b<1$. The formula for $\hat{J}$ from (30) gives

$$
\hat{J}(u, v)=f\left(\frac{\|u\|^{2}}{\|u\|_{4}^{2}}, \frac{\|v\|_{\omega}^{2}}{\|v\|_{4}^{2}}, \frac{b\|u v\|_{2}^{2}}{\|u\|_{4}^{2}\|v\|_{4}^{2}}\right)
$$

where the function

$$
f(x, y, z):= \begin{cases}\frac{x^{2}+y^{2}-2 z x y}{1-z^{2}}, & z<\min \left\{\frac{x}{y}, \frac{y}{x}\right\}, \\ \max \left\{x^{2}, y^{2}\right\}, & z \geq \min \left\{\frac{x}{y}, \frac{y}{x}\right\}\end{cases}
$$


is differentiable in $\left\{(x, y, z) \in \mathbb{R}_{>0} \times \mathbb{R}_{>0} \times[0,1): x \neq y\right\}$ with

$$
\begin{aligned}
& \nabla f(x, y, z)=(0,2 y, 0) \quad \text { if } 1>z \geq \frac{x}{y}, \\
& \nabla f(x, y, z)=(2 x, 0,0) \quad \text { if } 1>z \geq \frac{y}{x} .
\end{aligned}
$$

Hence, $\hat{J}$ is differentiable at $(\tilde{u}, \tilde{v})$. Since $(\tilde{u}, \tilde{v})$ minimizes $\hat{J}$ we get $\hat{J}^{\prime}(\tilde{u}, \tilde{v})=0$. In case $\frac{\|\tilde{v}\|_{\omega}}{\|\tilde{v}\|_{4}}>\frac{\|\tilde{u}\|_{1}}{\|\tilde{u}\|_{4}}$ this implies that the nonnegative function $\tilde{v}$ is a critical point of the functional $w \mapsto \frac{\|w\|_{\omega}^{2}}{\|w\|_{4}^{2}}$ and thus $-\Delta \tilde{v}+\omega^{2} \tilde{v}=\lambda \tilde{v}^{3}$ in $\mathbb{R}^{n}$ for some $\lambda>0$. Kwong's uniqueness result [8] then implies that $\tilde{v}$ is a translate of $\lambda^{-1 / 2} v_{0}$. Translating $(\tilde{u}, \tilde{v})$ if necessary we may assume $\tilde{v}=\lambda^{-1 / 2} v_{0}$. Since $(\tilde{u}, \tilde{v})$ does not satisfy condition (28) we obtain

$$
b=\frac{b\left\|\tilde{u} v_{0}\right\|_{2}^{2}}{\|\tilde{u}\|_{4}^{2}\left\|v_{0}\right\|_{4}^{2}} \cdot \frac{\|\tilde{u}\|_{4}^{2}\left\|v_{0}\right\|_{4}^{2}}{\left\|\tilde{u} v_{0}\right\|_{2}^{2}} \geq \frac{\|\tilde{u}\|^{2}\left\|v_{0}\right\|_{4}^{2}}{\|\tilde{u}\|_{4}^{2}\left\|v_{0}\right\|_{\omega}^{2}} \cdot \frac{\|\tilde{u}\|_{4}^{2}\left\|v_{0}\right\|_{4}^{2}}{\left\|\tilde{u} v_{0}\right\|_{2}^{2}}=\frac{\|\tilde{u}\|^{2}}{\left\|\tilde{u} v_{0}\right\|_{2}^{2}} \stackrel{(17)}{\geq} \underline{\mu}(\omega, n)
$$

which contradicts $b<\underline{\mu}(\omega, n)$. In case $\frac{\|\tilde{u}\|_{1}}{\|\tilde{u}\|_{4}}>\frac{\|\tilde{v}\|_{\omega}}{\|\tilde{v}\|_{4}}$ the analogous reasoning gives, up to translation, $\tilde{u}=\mu^{-1} u_{0}$ for some $\mu>0$ and thus

$$
\frac{\|\tilde{v}\|_{\omega}}{\|\tilde{v}\|_{4}}<\frac{\|\tilde{u}\|_{1}}{\|\tilde{u}\|_{4}}=\frac{\left\|u_{0}\right\|}{\left\|u_{0}\right\|_{4}}=\hat{c}_{0}
$$

which contradicts (12). In total we obtain that $(\tilde{u}, \tilde{v})$ must satisfy condition (28) which is all we had to show.

Proof of Theorem 3, part (ii):

Let $\mu(\omega, n) \leq b<1$. Then Proposition 5 gives $\left(0, v_{0}\right) \in \overline{\mathcal{M}_{b}}$ and (14) implies $\kappa_{b} \leq I\left(0, v_{0}\right)$. On the other hand we have $\left.I\right|_{\mathcal{M}_{b}}>I\left(0, v_{0}\right)$ which follows precisely as in the proof of (27). Both inequalities together give $\kappa_{b}=I\left(0, v_{0}\right)$ and that $\kappa_{b}$ is not attained.

\section{Proof of Theorem 3, part (iii):}

Let $\left(b_{j}\right)$ be a sequence converging to $\mu(\omega, n)$ from the left and let $\left(u_{j}, v_{j}\right)$ be a sequence of nonnegative minimizers of $\left.I\right|_{\mathcal{M}_{b}}$. The first claim will be proved once we show that $\left(u_{j}, v_{j}\right)$ has a subsequence which converges to $\left(0, v_{0}\right)$. As has been outlined in Sect. 2 the function $\left(u_{j}, v_{j}\right)$ is a positive radially symmetric and radially decreasing solution of (2) for $b$ replaced $b_{j}$. By (11) the sequence $\left(u_{j}, v_{j}\right)$ is bounded in $H^{1}\left(\mathbb{R}^{n}\right) \times H^{1}\left(\mathbb{R}^{n}\right)$ and has a subsequence, again denoted by $\left(u_{j}, v_{j}\right)$, which converges weakly, in $L^{4}\left(\mathbb{R}^{n}\right) \times L^{4}\left(\mathbb{R}^{n}\right)$ and almost everyhere to a nonnegative solution $(u, v)$ of (2) for $b:=\mu(\omega, n)$. Using these properties of the sequence $\left(u_{j}, v_{j}\right)$ as well as $\|u\|^{2}=\|u\|_{4}^{4}+b\|u v\|_{2}^{2},\|v\|_{\omega}^{2}=\|v\|_{4}^{4}+b\|u v\|_{2}^{2}$ we infer $\left\|u_{j}\right\| \rightarrow\|u\|,\left\|v_{j}\right\|_{\omega} \rightarrow\|v\|_{\omega}$ and thus $\left(u_{j}, v_{j}\right) \rightarrow(u, v)$ in $H^{1}\left(\mathbb{R}^{n}\right) \times$ $H^{1}\left(\mathbb{R}^{n}\right)$ as $j \rightarrow \infty$. From Proposition 4 and part (ii) we get

$$
I(u, v)=\lim _{j \rightarrow \infty} I\left(u_{j}, v_{j}\right)=\lim _{j \rightarrow \infty} \kappa_{b_{j}}=\kappa_{\underline{\mu}(\omega, n)}=I\left(0, v_{0}\right) .
$$

In particular, we have $(u, v) \neq(0,0)$ and the inequality $\left.I\right|_{\mathcal{M}_{b}}>I\left(0, v_{0}\right)$ from part (ii) implies $(u, v) \notin \mathcal{M}_{b}$. Hence, $(u, v)$ is a semitrivial nonnegative solution of (2) so that (34), (14) and $\omega>1$ implies $(u, v)=\left(0, v_{0}\right)$. 
Now set $\phi_{j}:=u_{j}\left\|u_{j}\right\|^{-1}$ for $j \in \mathbb{N}$ and it suffices to show $\phi_{j} \rightarrow \varphi$ for a suitable subsequence. Every $\phi_{j}$ is radially symmetric and radially decreasing and $\left(\phi_{j}\right)$ is bounded in $H^{1}\left(\mathbb{R}^{n}\right)$. Hence, we have $\phi_{j} \rightarrow \phi$ for some nonnegative $\phi \in H^{1}\left(\mathbb{R}^{n}\right)$ and hence $\phi_{j} \rightarrow \phi$ in $L^{4}\left(\mathbb{R}^{n}\right)$. From $\left\|u_{j}\right\|^{2}=\left\|u_{j}\right\|_{4}^{4}+b_{j}\left\|u_{j} v_{j}\right\|_{2}^{2}$ and $\left(u_{j}, v_{j}\right) \rightarrow\left(0, v_{0}\right)$ we infer

$$
\begin{aligned}
1 & =\frac{\left\|u_{j}\right\|_{4}^{4}+b_{j}\left\|u_{j} v_{j}\right\|_{2}^{2}}{\left\|u_{j}\right\|^{2}} \\
& =\left\|\phi_{j}\right\|_{4}^{2}\left\|u_{j}\right\|_{4}^{2}+b_{j}\left\|\phi_{j} v_{j}\right\|_{2}^{2} \\
& =o(1)+\underline{\mu}(\omega, n)\left\|\phi v_{0}\right\|_{2}^{2}
\end{aligned}
$$

which implies $\phi \neq 0$. Moreover, $\left\|\phi_{j}\right\|=1$ and $\phi_{j} \rightarrow \phi$ in $L^{4}\left(\mathbb{R}^{n}\right)$ gives

$$
\underline{\mu}(\omega, n) \stackrel{(17)}{\leq} \frac{\|\phi\|^{2}}{\left\|\phi v_{0}\right\|_{2}^{2}} \leq \lim _{j \rightarrow \infty} \frac{\left\|\phi_{j}\right\|^{2}}{\left\|\phi_{j} v_{0}\right\|_{2}^{2}}=\frac{1}{\left\|\phi v_{0}\right\|_{2}^{2}} \stackrel{(35)}{=} \underline{\mu}(\omega, n) .
$$

This implies $\left\|\phi_{j}\right\| \rightarrow\|\phi\|$ and hence $\phi_{j} \rightarrow \phi,\|\phi\|=1$ so that $\phi$ is the first eigenfunction of $(-\Delta+1)^{-1}\left(v_{0}^{2} \cdot\right)$ with $\|\phi\|=1$ and $\phi \geq 0$, hence $\phi=\varphi$. This proves $\phi_{j} \rightarrow \varphi$ as $j \rightarrow \infty$.

\section{Appendix A}

In this section we first determine all positive vector ground states of (2) and thus all nontrivial minimizers of $\left.I\right|_{\mathcal{N}_{b}}$ in the special case $\omega=1$. According to Remark 1 (a) we only need to consider parameters $b$ and $q$ satisfying

$$
1<q<2, \quad b>0 \quad \text { or } \quad q \geq 2, \quad b \geq 2^{q-1}-1 .
$$

In the special case $1<q<2,0<b<q-1$ we set $\mu_{b}:=\left(1+b k_{b}^{q}\right)^{-\frac{1}{2 q-2}}$ where $k_{b} \in(0,1)$ denotes the unique solution of $1+b k^{q}-b k^{q-2}-k^{2 q}=0$ in $(0,1)$.

Lemma 4. Let $n \in \mathbb{N}, 1<q<\frac{n}{(n-2)_{+}}, \omega=1$ and let $b, q$ satisfy (36). Then all positive vector ground states of (2) are translates of the following functions:

$$
\begin{array}{ll}
\left(\mu_{b} k_{b} u_{0}, \mu_{b} u_{0}\right) \text { or }\left(\mu_{b} u_{0}, \mu_{b} k_{b} u_{0}\right) & \text { in case } 1<q<2,0<b<q-1, \\
(1+b)^{-\frac{1}{2 q-2}}\left(u_{0}, u_{0}\right) & \text { in case } 1<q<2, b \geq q-1, \\
\left(\cos (\alpha) u_{0}, \sin (\alpha) u_{0}\right) \quad\left(0<\alpha<\frac{\pi}{2}\right) & \text { in case } q=2, b=1 \\
(1+b)^{-\frac{1}{2 q-2}}\left(u_{0}, u_{0}\right) & \text { in case } q=2, b>1, \\
(1+b)^{-\frac{1}{2 q-2}}\left(u_{0}, u_{0}\right) & \text { in case } q>2, b \geq 2^{q-1}-1 .
\end{array}
$$


Proof. Let $(u, v) \in \mathcal{N}_{b}$ be a positive function. Then the following holds

$$
\begin{aligned}
I(u, v) & =\frac{q-1}{2 q}\left(\frac{\left(\|u\|^{2}+\|v\|^{2}\right)^{q}}{\|u\|_{2 q}^{2 q}+\|v\|_{2 q}^{2 q}+2 b\|u v\|_{q}^{q}}\right)^{\frac{1}{q-1}} \\
& \stackrel{(12)}{\geq} \frac{q-1}{2 q}\left(\frac{\left(\hat{c}_{0}^{2}\|u\|_{2 q}^{2}+\hat{c}_{0}^{2}\|v\|_{2 q}^{2}\right)^{q}}{\|u\|_{2 q}^{2 q}+\|v\|_{2 q}^{2 q}+2 b\|u\|_{2 q}^{q}\|v\|_{2 q}^{q}}\right)^{\frac{1}{q-1}} \\
& \geq\left(\inf _{k>0} \frac{\left(1+k^{2}\right)^{q}}{1+k^{2 q}+2 b k^{q}}\right)^{\frac{1}{q-1}} \cdot \frac{q-1}{2 q} \hat{c}_{0}^{\frac{2 q}{q-1}} \\
& \stackrel{(13)}{=}\left(\inf _{k>0} \frac{\left(1+k^{2}\right)^{q}}{1+k^{2 q}+2 b k^{q}}\right)^{\frac{1}{q-1}} \cdot I\left(u_{0}, 0\right) .
\end{aligned}
$$

Here equality holds if and only if $(u, v)$ is a translate of $\left(\lambda u_{0}, \mu u_{0}\right)$ where $\lambda, \mu>$ 0 and $k^{*}:=\mu \lambda^{-1}$ minimizes the function $k \mapsto\left(1+k^{2}\right)^{q}\left(1+k^{2 q}+2 b k^{q}\right)^{-1}$ over $(0, \infty)$. In case $1<q<2$ and $0<b<q-1$ this implies $k^{*}=k_{b}$ or $k^{*}=k_{b}^{-1}$ and $(u, v) \in \mathcal{N}_{b}$ gives $(u, v)=\left(\mu_{b} k_{b} u_{0}, \mu_{b} u_{0}\right)$ or $(u, v)=\left(\mu_{b} u_{0}, \mu_{b} k_{b} u_{0}\right)$. In all other cases except $q=2, b=1$ a similar discussion leads to $k^{*}=1$ and thus $(u, v)=(1+b)^{-\frac{1}{2 q-2}}\left(u_{0}, u_{0}\right)$. In case $q=2, b=1$ we find that $k^{*}$ is arbitrary which results in $(u, v)=\left(\cos (\alpha) u_{0}, \sin (\alpha) u_{0}\right)$ for some $\alpha \in\left(0, \frac{\pi}{2}\right)$.

Finally we determine the minimizers of the functional $\left.I\right|_{\mathcal{M}_{b}}$ when $\omega=1$. By Lemma 3 we may restrict ourselves to the case $q \geq 2, b \geq 0$. We will make use of the inequality

$$
\frac{\min \left\{1+b r^{q}, r^{2 q-2}+b r^{q-2}\right\}}{\left(1+r^{2}\right)^{q-1}} \leq \frac{1+b}{2^{q-1}} \quad(b \geq 0, r>0)
$$

and the additional information that in case $q>2$ or $q=2, b \neq 1$ equality is achieved only at $r=1$. For a proof of this technical result we refer to [12], p. 132 .

Lemma 5. Let $n \in\{1,2,3\}, 2 \leq q<\frac{n}{(n-2)_{+}}$and $\omega=1, b \geq 0$. All positive minimizers of $\left.I\right|_{\mathcal{M}_{b}}$ are translates of

$$
\begin{aligned}
& (1+b)^{-\frac{1}{2 q-2}}\left(u_{0}, u_{0}\right) \\
& \left(\cos (\alpha) u_{0}, \sin (\alpha) u_{0}\right) \quad\left(0<\alpha<\frac{\pi}{2}\right) \quad \text { in case } q=2, b=1 \text {, } \\
& (1+b)^{-\frac{1}{2 q-2}}\left(u_{0}, u_{0}\right) \quad \text { in case } q>2, b \geq 0 .
\end{aligned}
$$

Proof. Let $(u, v) \in \mathcal{M}_{b}$ be a positive function and set $r:=\|v\|_{2 q}\|u\|_{2 q}^{-1}$. From $\|u\|^{2}=\|u\|_{2 q}^{2 q}+b\|u v\|_{q}^{q},\|v\|^{2}=\|v\|_{2 q}^{2 q}+b\|u v\|_{q}^{q}$ and Hölder's inequality we get $I(u, v)=\frac{q-1}{2 q}\left(\|u\|^{2}+\|v\|^{2}\right)$

$$
\begin{aligned}
& \stackrel{(12)}{\geq} \frac{q-1}{2 q} \hat{c}_{0}^{2}\left(\|u\|_{2 q}^{2}+\|v\|_{2 q}^{2}\right) \\
& \stackrel{(37)}{\geq} \frac{q-1}{2 q} \hat{c}_{0}^{2}\left(\|u\|_{2 q}^{2}+\|v\|_{2 q}^{2}\right) \cdot\left(\frac{2^{q-1}}{1+b} \cdot \frac{\min \left\{1+b r^{q}, r^{2 q-2}+b r^{q-2}\right\}}{\left(1+r^{2}\right)^{q-1}}\right)^{\frac{1}{q-1}} .
\end{aligned}
$$


Using $r=\|v\|_{2 q}\|u\|_{2 q}^{-1}$ and

$$
\begin{gathered}
=\frac{q-1}{q} \hat{c}_{0}^{2}(1+b)^{-\frac{1}{q-1}}\|u\|_{2 q}^{2} \min \left\{1+b r^{q}, r^{2 q-2}+b r^{q-2}\right\}^{\frac{1}{q-1}} \\
\|u\|^{2}=\|u\|_{2 q}^{2 q}+b\|u v\|_{q}^{q} \leq\|u\|_{2 q}^{2 q}\left(1+b r^{q}\right) \\
\|v\|^{2}=\|v\|_{2 q}^{2 q}+b\|u v\|_{q}^{q} \leq\|u\|_{2 q}^{2 q-2}\|v\|_{2 q}^{2}\left(r^{2 q-2}+b r^{q-2}\right)
\end{gathered}
$$

we infer

$$
\begin{aligned}
I(u, v) & \geq \frac{q-1}{q} \hat{c}_{0}^{2}(1+b)^{-\frac{1}{q-1}} \min \left\{\frac{\|u\|^{2}}{\|u\|_{2 q}^{2}}, \frac{\|v\|^{2}}{\|v\|_{2 q}^{2}}\right\}^{\frac{1}{q-1}} \\
& \stackrel{(12)}{\geq} \frac{q-1}{q} \hat{c}_{0}^{\frac{2 q}{q-1}}(1+b)^{-\frac{1}{q-1}} \\
& \stackrel{(13)}{=} 2(1+b)^{-\frac{1}{q-1}} I\left(u_{0}, 0\right) .
\end{aligned}
$$

Here, equality holds if and only if $(u, v)$ is a translate of $\left(\lambda u_{0}, \mu u_{0}\right)$ and $r=$ $\mu \lambda^{-1}$ satisfies

$$
\frac{\min \left\{1+b r^{q}, r^{2 q-2}+b r^{q-2}\right\}}{\left(1+r^{2}\right)^{q-1}}=\frac{1+b}{2^{q-1}} .
$$

In the case $q=2, b=1$ this does not respresent a restriction and we obtain $(u, v)=\left(\cos (\alpha) u_{0}, \sin (\alpha) u_{0}\right)$ for some $0<\alpha<\frac{\pi}{2}$. In all other cases, however, this implies $r=1$ and thus $(u, v)=(1+b)^{-\frac{1}{2 q-2}}\left(u_{0}, u_{0}\right)$.

\section{Appendix B}

In this final section we discuss the borderline case $b=b(\omega, q, n)$ in Theorem 1. First we answer the question whether vector ground states exist for $b=b(\omega, q, n)$ in the case $q \neq 2$.

Lemma 6. Let $n \in \mathbb{N}, 1<q<\frac{n}{(n-2)_{+}}$and $\omega \geq 1$.

(i) In case $1<q<2, b=b(\omega, q, n)=0$ no vector ground state exists.

(ii) In case $q>2, b=b(\omega, q, n)$ a vector ground state exists.

In both cases we have $c_{b}=I\left(u_{0}, 0\right)$.

Proof. Let $b=0$. Since $u_{0}$ and $v_{0}$ are the minimal energy solutions of the scalar equations associated to (2) we obtain that a nonnegative ground state is, up to translation, given by $\left(u_{0}, 0\right)$ or $\left(0, v_{0}\right)$ or $\left(u_{0}, v_{0}\right)$. From $I\left(u_{0}, v_{0}\right)=$ $I\left(u_{0}, 0\right)+I\left(0, v_{0}\right)>I\left(0, v_{0}\right) \geq I\left(u_{0}, 0\right)$ we obtain that $\left(u_{0}, 0\right)$ minimizes $\left.I\right|_{\mathcal{N}_{b}}$ which proves (i).

Now let $q>2$ and let $\left(b_{j}\right)$ be a sequence that converges to $b:=b(\omega, q, n)$ from the right. The corresponding radially symmetric and radially decreasing 
vector ground states will be denoted by $\left(u_{j}, v_{j}\right)$. From $\left(u_{j}, v_{j}\right) \in \mathcal{M}_{b_{j}}$ and Sobolev's inequality we infer

$$
\begin{aligned}
\left\|u_{j}\right\|_{2 q}^{2} & \leq c\left\|u_{j}\right\|^{2}=c\left(\left\|u_{j}\right\|_{2 q}^{2 q}+b_{j}\left\|u_{j} v_{j}\right\|_{q}^{q}\right) \\
& \leq c(1+b+o(1))\left\|u_{j}\right\|_{2 q}^{q}\left(\left\|u_{j}\right\|_{2 q}^{q}+\left\|v_{j}\right\|_{2 q}^{q}\right) \\
\left\|v_{j}\right\|_{2 q}^{2} & \leq c\left\|v_{j}\right\|_{\omega}^{2}=c\left(\left\|v_{j}\right\|_{2 q}^{2 q}+b_{j}\left\|u_{j} v_{j}\right\|_{2 q}^{q}\right) \\
& \leq c(1+b+o(1))\left\|v_{j}\right\|_{2 q}^{q}\left(\left\|u_{j}\right\|_{2 q}^{q}+\left\|v_{j}\right\|_{2 q}^{q}\right)
\end{aligned}
$$

for some positive number $c>0$ so that $q>2$ implies $\tilde{c} \leq\left\|u_{j}\right\|_{2 q},\left\|v_{j}\right\|_{2 q} \leq$ $\tilde{c}^{-1}$ for some $\tilde{c}>0$. Here we used that the sequence $\left(u_{j}, v_{j}\right)$ is bounded in $H^{1}\left(\mathbb{R}^{n}\right) \times H^{1}\left(\mathbb{R}^{n}\right)$ and thus in $L^{2 q}\left(\mathbb{R}^{n}\right) \times L^{2 q}\left(\mathbb{R}^{n}\right)$. We may pass to a weakly convergent subsequence $\left(u_{j}, v_{j}\right)$ which converges in $L^{2 q}\left(\mathbb{R}^{n}\right) \times L^{2 q}\left(\mathbb{R}^{n}\right)$ to its weak limit $(u, v)$. Since $(u, v)$ solves (2) we have $\|u\|^{2}=\|u\|_{2 q}^{2 q}+b\|u v\|_{q}^{q}$, $\|v\|_{\omega}^{2}=\|v\|_{2 q}^{2 q}+b\|u v\|_{q}^{q}$ which gives $\left(u_{j}, v_{j}\right) \rightarrow(u, v)$ in $H^{1}\left(\mathbb{R}^{n}\right) \times H^{1}\left(\mathbb{R}^{n}\right)$. The above estimates for $\left\|u_{j}\right\|_{2 q},\left\|v_{j}\right\|_{2 q}$ show that $(u, v)$ is fully nontrivial and hence $(u, v)$ is a vector ground state due to

$$
I(u, v)=\lim _{j \rightarrow \infty} I\left(u_{j}, v_{j}\right)=\lim _{j \rightarrow \infty} c_{b_{j}}=c_{b} .
$$

Finally let us mention that in the case of the cubic nonlinearity it is not known whether vector ground states exist for $b=b(\omega, 2, n)$. If one could prove $b(\omega, 2, n)<\bar{\mu}(\omega, n)$ for some parameters $\omega, n$ then the vector ground states $\left(u_{j}, v_{j}\right)$ associated to coupling parameters $b_{j}>b(\omega, q, n)$ would not converge to the semitrivial solution $\left(u_{0}, 0\right)$ but to a vector ground state as $b_{j} \rightarrow b(\omega, q, n)$. In the other case $b(\omega, 2, n)=\bar{\mu}(\omega, n)$ one would expect that $\left(u_{j}, v_{j}\right)$ converges to $\left(u_{0}, 0\right)$ so that bifurcation from the semitrivial solution branch $\left\{\left(u_{0}, 0, b\right): b \in \mathbb{R}\right\}$ occurs at $b=\bar{\mu}(\omega, n)$. The question which of these alternative applies is particularly interesting. Unfortunately, we have to leave it unanswered.

\section{References}

[1] Ambrosetti, A., Colorado, E.: Bound and ground states of coupled nonlinear Schrödinger equations. C. R. Math. Acad. Sci. Paris 342(7), 453-458 (2006)

[2] Bartsch, T., Dancer, N., Wang, Z.-Q.: A Liouville theorem, a-priori bounds, and bifurcating branches of positive solutions for a nonlinear elliptic system. Calc. Var. Partial Differ. Equ. 37(3-4), 345-361 (2010)

[3] Busca, J., Sirakov, B.: Symmetry results for semilinear elliptic systems in the whole space. J. Differ. Equ. 163(1), 41-56 (2000)

[4] Chen, Z., Zou, W.: An optimal constant for the existence of least energy solutions of a coupled Schrödinger system. Calc. Var. Partial Differ. Equ. 48(3-4), 695711. doi:10.1007/s00526-012-0568-2 
[5] de Figueiredo, D.G., Lopes, O.: Solitary waves for some nonlinear Schrödinger systems. Ann. Inst. H. Poincaré Anal. Non Linéaire 25(1), 149-161 (2008)

[6] Hundertmark, D.: Some bound state problems in quantum mechanics. In: Spectral Theory and Mathematical Physics: A Festschrift in Honor of Barry Simon's 60th Birthday, Volume 76 of Proc. Sympos. Pure Math., pp. 463-496. American Mathematical Society, Providence(2007)

[7] Ikoma, N.: Uniqueness of positive solutions for a nonlinear elliptic system. NoDEA Nonlinear Differ. Equ. Appl. 16(5), 555-567 (2009)

[8] Kwong, M.K.: Uniqueness of positive solutions of $\Delta u-u+u^{p}=0$ in $R^{n}$. Arch. Rational Mech. Anal. 105(3), 243-266 (1989)

[9] Lin, T.-C., Wei, J.: Ground state of $N$ coupled nonlinear Schrödinger equations in $\mathbb{R}^{n}, n \leq 3$. Commun. Math. Phys. 255(3), 629-653 (2005)

[10] Lin, T.-C., Wei, J.: Erratum: "Ground state of $N$ coupled nonlinear Schrödinger equations in $\mathbb{R}^{n}, n \leq 3$ " [Comm. Math. Phys. 255 (2005), no. 3, 629-653]. Commun. Math. Phys. 277(2):573-576 (2008)

[11] Maia, L.A., Montefusco, E., Pellacci, B.: Positive solutions for a weakly coupled nonlinear Schrödinger system. J. Differ. Equ. 229(2), 743-767 (2006)

[12] Mandel, R.: Grundzustände, Verzweigungen und singuläre Lösungen nichtlinearer Schrödingersysteme. PhD thesis, Karlsruhe Institute of Technology (KIT) (2013)

[13] Mandel, R.: Minimal energy solutions for repulsive nonlinear schrödinger systems. J. Differ. Equ. 257(2), 450-468 (2014). doi:10.1016/j.jde.2014.04.006

[14] Sirakov, B.: Least energy solitary waves for a system of nonlinear Schrödinger equations in $\mathbb{R}^{n}$. Commun. Math. Phys. 271(1), 199-221 (2007)

[15] Wei, J., Weth, T.: Nonradial symmetric bound states for a system of coupled Schrödinger equations. Atti Accad. Naz. Lincei Cl. Sci. Fis. Mat. Natur. Rend. Lincei (9) Mat. Appl. 18(3), 279-293 (2007)

Rainer Mandel

Department of Mathematics

Karlsruhe Institute of Technology (KIT)

76128 Karlsruhe

Germany

e-mail: Rainer.Mandel@kit.edu

Received: 13 February 2014.

Accepted: 16 June 2014. 